\title{
High-Mobility Bismuth-based Transparent P-Type Oxide from High-throughput Material Screening
}

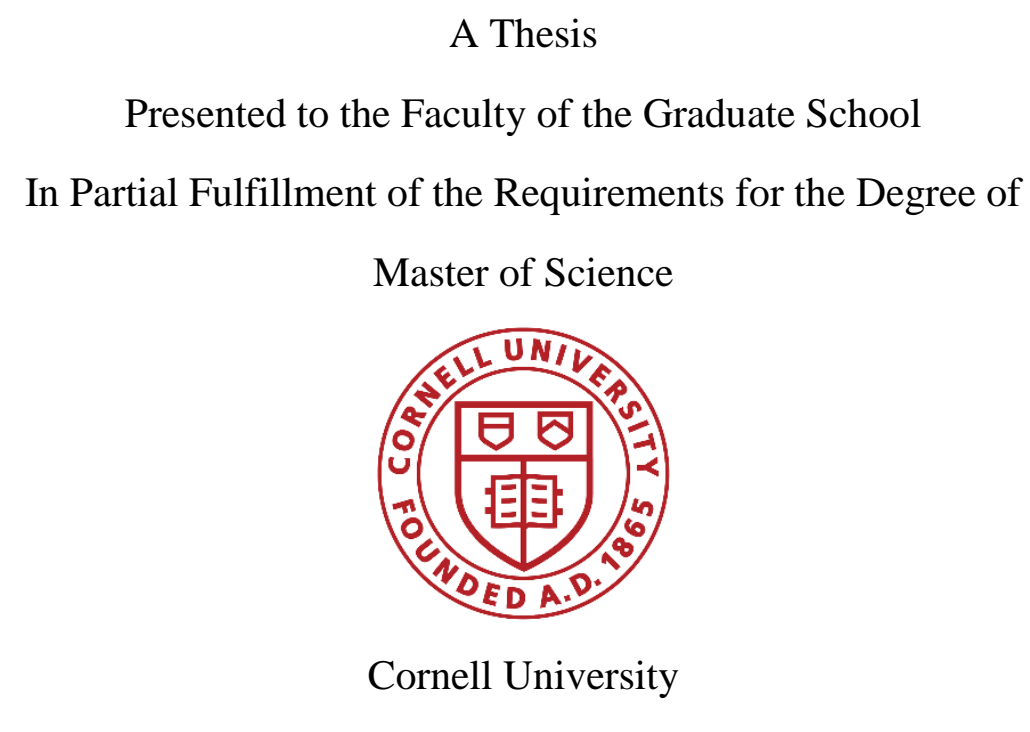

By

Amit Bhatia

August 2015 
(C) 2015 Amit Bhatia 


\begin{abstract}
Transparent conducting oxides (TCOs) have gathered widespread attention in the last few decades. This can be attributed to TCOs being an essential building block to many technologies, ranging from components in transparent and flexible electronics ${ }^{1,2}$, transparent conductors ${ }^{3,4}$, to absorbers and protection layers in photovoltaics and photoelectrochemical devices ${ }^{5,6}$. However, thus far, it has been difficult to develop p-type oxides with wide band gap and high hole mobility; current state-of-art transparent p-type oxides have hole mobility in the range of $<10 \mathrm{~cm}^{2} / \mathrm{V} \cdot \mathrm{s}^{7,8}$, much lower than their n-type counterparts ${ }^{9-11}$. We show the experimental synthesis, optical and electrical characterization of a novel bismuth-based double-perovskite oxide with wide band gap and high hole mobility found as a result of recent high-throughput computational screening ${ }^{12}$. Our candidate, $\mathrm{Ba}_{2} \mathrm{BiTaO}_{6}$, has an optical band gap larger than $4 \mathrm{eV}$ and a Hall hole mobility above 30 $\mathrm{cm}^{2} / \mathrm{V} \cdot \mathrm{s}$. We rationalize this finding with molecular orbital intuitions; $\mathrm{Bi}^{3+}$ with filled s-orbitals strongly overlap with the oxygen $\mathrm{p}$, increasing the extent of the metal-oxygen covalency and effectively reducing the valence effective mass, while $\mathrm{Ta}^{5+}$ forms a conduction band with low electron negativity, leading to a high band gap beyond the visible range. These results lead to introduction of a completely new class of Bi based p-type transparent conducting oxides which have never been considered before. Our concerted theory-experiment effort points to the growing utility of a data-driven materials discovery and the combination of both informatics and chemical intuitions as a way to discover future technological materials.
\end{abstract}


To my parents, Savita and Harish Bhatia, my fiancée, Jaspreet and my teachers 


\section{Acknowledgments}

To describe my experience at Cornell as merely positive would be an understatement. As I think of my two years spent here, I am overwhelmed with a sense of gratitude, accomplishment and personal growth that would not have been possible without the help and support of so many people.

First and foremost, I must acknowledge my advisor Prof. Jin Suntivich for giving me an opportunity to start this project and it would not have been possible to finish it without his mentorship. His vision, intelligence and hard work never cease to surprise me. I have always considered joining The Suntivich group as the best decision of my career till now and there has been no single day when I didn't learn something new. I am extremely thankful to Jin for supporting me throughout my endeavors here and for showing remarkable patience, when I admittedly struggled to achieve the level of proficiency required to succeed as a graduate student. I must admit that there were numerous occasions when I thought why Jin accepted me to his group, but I am glad he stuck with me, as he has been a constant source of motivation and it has only been through his encouragement, enthusiasm and optimism that I matured as a researcher and my love for science (and semiconductors) grew.

I must also acknowledge my thesis committee member, Prof. David Muller, who on numerous occasions provided me with out of the box solutions to the problems I faced while working on the project; Prof. Geoffroy Hautier, without whose fruitful collaboration with Jin, this project would not have started in the first place; Dr. Christopher Evans, who has been like a second advisor to me, always approachable, willing to discuss anything and everything, and had faith in me more than anyone else.

Furthermore, I would also like to express my special thanks to Tan, who I have had the pleasure of mentoring over the past one year, worked with me on this project and did the mobility 
measurements, John, my fellow graduate student, for inspiring me with his clear thinking and rational attitude, Anielli, Chengyu, Runbang and Avik, with whom I have enjoyed great friendship. I am honored to have been part of such a collaborative group of talented researchers.

I would love to thank Phil and Maura for teaching me how to use the experimental facilities and Cornell Center for Materials Research for allowing to use their shared facilities and resources.

Last, but definitely not the least, I am grateful to my parents, who have always supported me for every decision that I have made and encouraged me to follow my heart and fulfill my academic dreams. Finally, to my love Jaspreet: thank you for your unwavering love and support. I couldn't have done this without you.

Amit Bhatia

May 2015 


\section{Table of Contents}

List of Figure

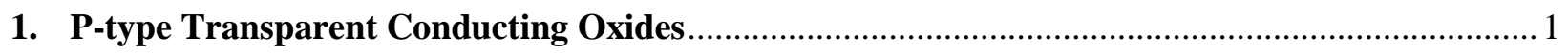

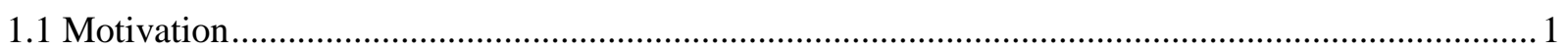

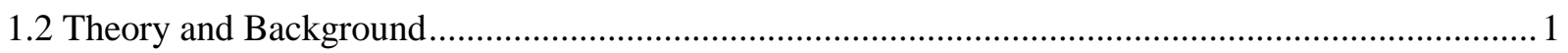

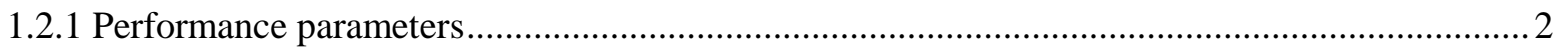

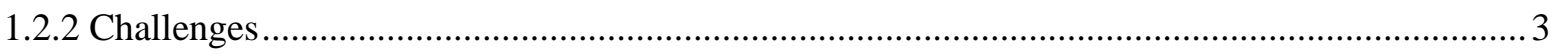

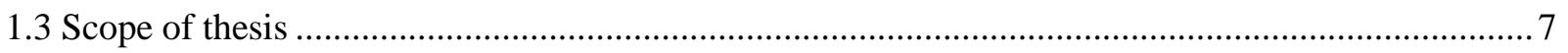

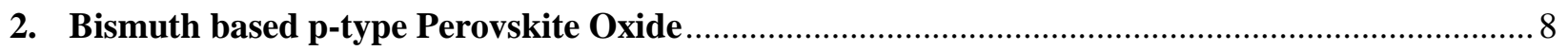

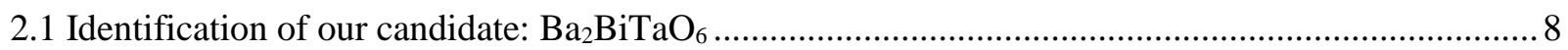

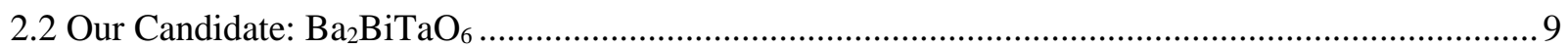

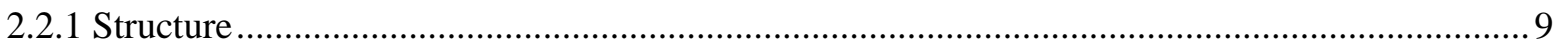

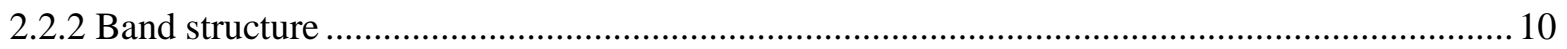

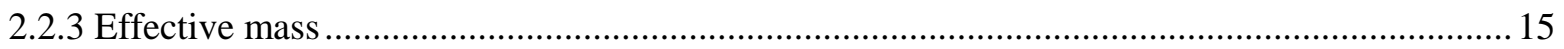

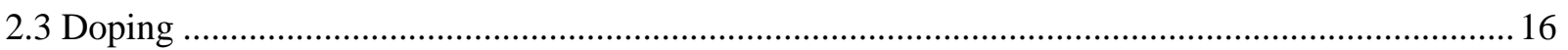

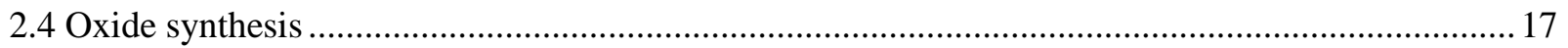

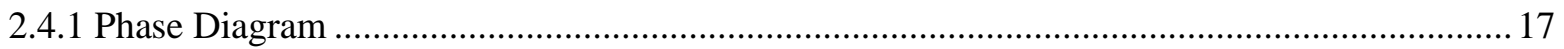

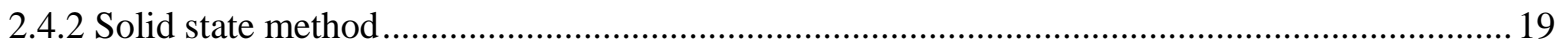

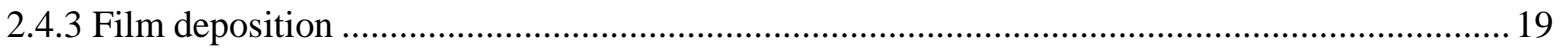

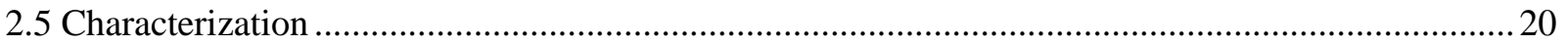

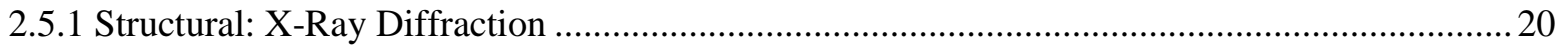

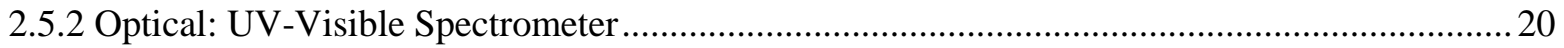

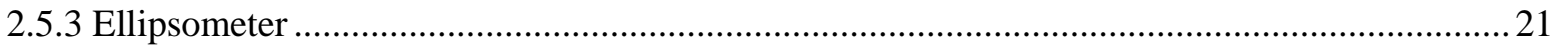

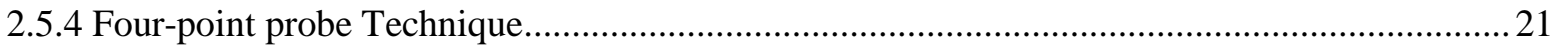

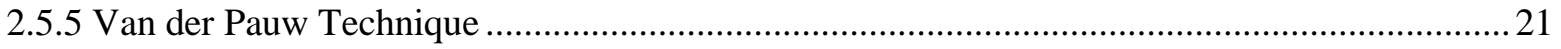

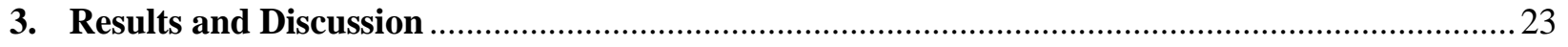

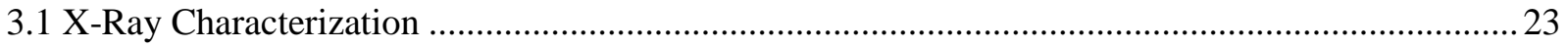

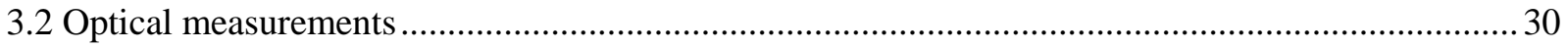

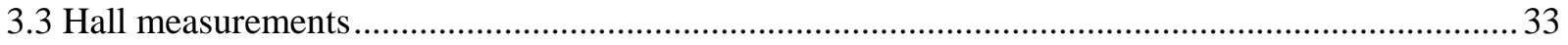

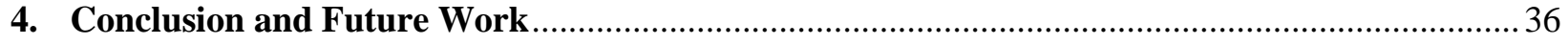

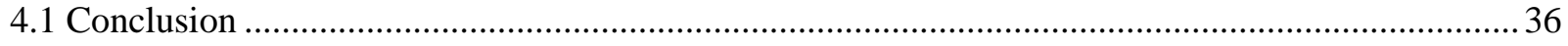

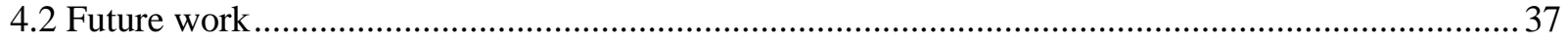


Bibliography 


\section{List of Figures}

Figure 1.1 Illustrates a comparison of majority charge carrier mobility (in $\mathrm{cm} 2 / \mathrm{V}$.s) vs band gap (in $\mathrm{eV}$ ) of a few state-of-the-art p-type (red) and n-type (blue) transparent conducting oxides.

Figure 1.2 Illustrates the relationship between band curvature and charge carrier effective mass (and hence, mobility).

Figure 2.1 Structure of BBT (a double perovskite oxide of the form $\mathrm{A}_{2} \mathrm{BB}^{\prime} \mathrm{O}_{6}$ ), where yellow atoms represent $\mathrm{Ba}^{2+}(\mathrm{A}$-site), and red atoms represent oxygen, and atoms at the center of purple and grey octahedra represent $\mathrm{Bi}^{3+}$ (B-site) and $\mathrm{Ta}^{5+}\left(\mathrm{B}^{\prime}\right.$-site) respectively.

Figure 2.2 Illustrates the Band structure of $\mathrm{Ba}_{2} \mathrm{BiTaO}_{6}$ along symmetry lines and density of state projected on different atomic sites using GGA. A scissor operator was applied to the band gap to make it fit the single-shot GW value.

Figure 2.3 Illustrates square of the wave function in DFT-GGA for the valence band in $\mathrm{BiO}_{6}$ octahedra.

Figure 2.4 Illustrates density of state from a projection on localized basis sets using the lobster software on GGA-PBE results, (center) COHP analysis, and (right) schematic molecular orbital diagram for the $\mathrm{Bi} 6 \mathrm{~s}-\mathrm{O} 2 \mathrm{p}$ hybridization.

Figure 2.5 Illustrates the conduction band and valence band levels vs vacuum for BBT compared to typical n-type TCOs $\left(\mathrm{In}_{2} \mathrm{O}_{3}\right.$ and $\left.\mathrm{ZnO}\right)$ as well as p-type TCOs $\left(\mathrm{CuAlO}_{2}\right.$ and $\mathrm{SnO})$.

Figure 2.6 Illustrates the comparison of majority charge carrier effective mass and band gap of state-of-the-art p-type and n-type TCO with BBT (computationally predicted).

Figure 2.7 Illustrates the phase diagram of barium oxide $(\mathrm{BaO})$, bismuth oxide $\left(\mathrm{Bi}_{2} \mathrm{O}_{3}\right)$ and tantalum oxide $\left(\mathrm{Ta}_{2} \mathrm{O}_{5}\right)$ utilized for synthesizing the pure phase chemistry. Picture adopted from materialsproject.org.

Figure 3.1 Illustrates the phase pure X-ray diffraction data of BBT synthesized at 1100C, 12 hrs in argon atmosphere.

Figure 3.2 Illustrates the X-Ray diffraction studies done on $\mathrm{Ba}_{2-\mathrm{x}} \mathrm{K}_{\mathrm{x}} \mathrm{BiTaO}_{6}(\mathrm{BKBT})$, where $\mathrm{x}$ $=(0-0.35)$. No extra secondary impurity phases were detected.

Figure 3.3 Illustrates the peak shifting as $\mathrm{K}$ is added as a substitutional impurity in place of $\mathrm{Ba}$ in BKBT. 
Figure 3.4 Bruker General Area Detector Diffraction System (GADDS) of $\mathrm{Ba}_{2} \mathrm{BiTaO}_{6}$ thin films.

Figure 3.5 2Theta-phi scan done on the $\mathrm{Ba}_{2} \mathrm{BiTaO}_{6}$ films deposited onto (100) $\mathrm{MgO}$ substrate.

Figure 3.6 Bruker General Area Detector Diffraction System (GADDS) of $\mathrm{Ba}_{1.3} \mathrm{~K}_{0.7} \mathrm{BiTaO}_{6}$ thin films.

Figure 3.7 2Theta-phi scan done on the $\mathrm{Ba}_{1.3} \mathrm{~K}_{0.7} \mathrm{BiTaO}_{6}$ films deposited onto (100) $\mathrm{MgO}$ substrate.

Figure 3.8 Transparency vs wavelength data from UV-Vis Spectrometer in wavelength range from $200-800 \mathrm{~nm}$ for BBT vs BKBT films. Transparency of the films was maintained well above $90 \%$ even after adding $\mathrm{K}=35 \%$ in BKBT.

Figure 3.9 Illustrates the $(\alpha h v)^{2}$ vs Energy plot of the $\mathrm{Ba}_{2-\mathrm{x}} \mathrm{K}_{\mathrm{x}} \mathrm{BiTaO}_{6}$ films grown on $\mathrm{MgO}$ (100) substrate. A rough estimate of direct band gap of the material can be made from this curve.

Figure 3.10 Illustrates the $(\alpha)^{0.5}$ vs Energy plot of $\mathrm{Ba}_{2-\mathrm{x}} \mathrm{K}_{\mathrm{x}} \mathrm{BiTaO}_{6}$ films grown on $\mathrm{MgO}(100)$ substrate. A rough estimate of indirect band gap of the material can be made from this curve.

Figure 3.11 Illustrates hall voltages as a function of magnetic field. The slope was used to estimate the carrier concentration, which was calculated to be $\sim 5 \times 10^{13} \mathrm{~cm}^{-3}$ (yielding the hole mobility of $\sim 33 \mathrm{~cm}^{2} / \mathrm{V} \bullet \mathrm{s}$ ). In a separate measurement on a sample synthesized independently, the carrier concentration was calculated to be $\sim 9 \times 10^{13}$ $\mathrm{cm}^{-3}$ (yielding the hole mobility of $\sim 35 \mathrm{~cm}^{2} / \mathrm{V} \cdot \mathrm{s}$ ).

Figure 4.1 Illustrates hall mobility $\left(\mathrm{cm}^{2} / \mathrm{V}-\mathrm{s}\right)$ vs band gap $(\mathrm{eV})$ of $\mathrm{Ba}_{2} \mathrm{BiTaO}_{6}$ in comparison with other existing state-of-the-art p-type transparent conducting oxides. 


\section{Chapter 1 - P-type Transparent Conducting Oxides}

\subsection{Motivation}

Transparent Conducting oxides have gathered widespread attention in the last few decades owing to their strong presence in large part of the electronic industry ranging from portable electronic displays, flexible electronics, multifunctional windows, solar cells ${ }^{13,14}$ and most recently transparent transistors ${ }^{15,16}$. Currently, the strongest demand for TCOs stems from the flat panel displays and energy efficient window applications, although with the rapidly growing PV industry, the demand for highly efficient TCOs is expected to grow exponentially in the near future. Many n-type TCOs with good carrier mobility and high optical transparency, such as $\mathrm{ZnO}$, In-Sn-O and In-Ga-Zn-O ${ }^{1,9,10,17}$, have already been in use. However, their p-type counterparts have struggled to achieve similar performances. Localization of the oxygen $2 p$ state in valence band is widely believed to be the reason behind the poor performance of $\mathrm{p}$-type TCOs ${ }^{18,19}$. The goal of this thesis is to assist in finding novel set of p-type oxides that have never been previously considered for the application of transparent conductors by unveiling the strategies to delocalize the valence band in this case by introduction of metal cation $\left(\mathrm{Bi}^{3+}\right)$ having energy level similar to $\mathrm{O} 2 p$ level.

\subsection{Theory and Background}

TCOs are a special class of materials that are both optically transparent as well as electrically conductive. Usually the optically transparent materials (band gap $>3.1 \mathrm{eV}$ ) tend to be electrically insulating by the virtue of large band gap. However, typical TCOs exhibit both transparency > $80 \%$ as well as electrical conductivity of the order of $10^{4} \mathrm{~S} / \mathrm{cm}$. 
In-Sn-O, popularly known as ITO is the most commonly used TCO in the industry. However, it has its own limitations of high cost, scarcity and highly brittle nature to be used on flexible substrates. Not only that, good quality ITO needs to be deposited at higher temperatures thereby limiting the choice of substrates. At present, the market for TCO has been expanding at a very high rate, thereby suggesting need for earth-abundant, non-toxic, cheap and environmental friendly TCO materials.

\subsubsection{Performance parameters}

The performance rating of a TCO is highly dependent on the application. Usually, two major properties define the basic performance requirement of a TCO: namely, optical transparency and electrical conductivity. The properties of a TCO are dependent a number of factors, a few important one being chemical composition, synthesis and deposition parameter.

Ideally, a TCO should be $100 \%$ transparent in the near UV, visible and near IR region. In other words, it should have a low absorption coefficient in the mentioned regions.

Since a TCO needs to have both high electrical conductivity and low optical absorption coefficient in the aforementioned regions, therefore, the performance of a TCO is rated by the ratio of electrical conductivity $\sigma$ and visible absorption coefficient $\alpha$. This ratio $\sigma / \alpha$ is known as the figureof-merit of a material. Higher is the value of figure-of-merit, better is the performance of a TCO. 
Figure-of-merit of a TCO is given as:

$$
\sigma / \alpha=-\left\{R_{s} \ln (T+R)\right\}^{-1}
$$

where $R_{s}$ is the sheet resistant in ohms per square, $T$ and $R$ being the total transmission and visible reflectance respectively ${ }^{20}$. The value of $\sigma$ and $\alpha$ depends on the grain size of the crystal. Hence, epitaxially deposited mono-crystalline materials have higher figure-of-merit as compared to the poly-crystalline materials with numerous grain boundaries. These grain boundaries have a tendency to increase both optical and electrical scattering thereby resulting in a high absorption coefficient and low electrical conductivity. Therefore, the deposition parameters such as temperature of deposition, ambient pressure etc. and choice of a suitable substrate play a crucial role in deciding the performance a TCO.

\subsubsection{Challenges}

One of the major challenge in front of TCO research community today is to find p-type materials with conductivities comparable to their n-type counterparts, i.e., of order $10^{3} \mathrm{~S} / \mathrm{cm}$. It has been widely believed that high hole effective mass, and hence low hole mobility, limits the performance of p-type TCOs. Figure 1.1 illustrates a comparison of band gap and majority charge carrier mobility of a few state-of-the-art TCOs (both n-type and p-type). The best p-type TCO $\left(\mathrm{CuAlO}_{2}\right)$ is known to have highest mobility $<10 \mathrm{~cm}^{2} / \mathrm{V} \cdot \mathrm{s}^{13,21-24}$, much lower than the n-type oxides (In-Sn$\left.\mathrm{O} \sim 100 \mathrm{~cm}^{2} / \mathrm{V}-\mathrm{s}\right)^{9-11}$ 


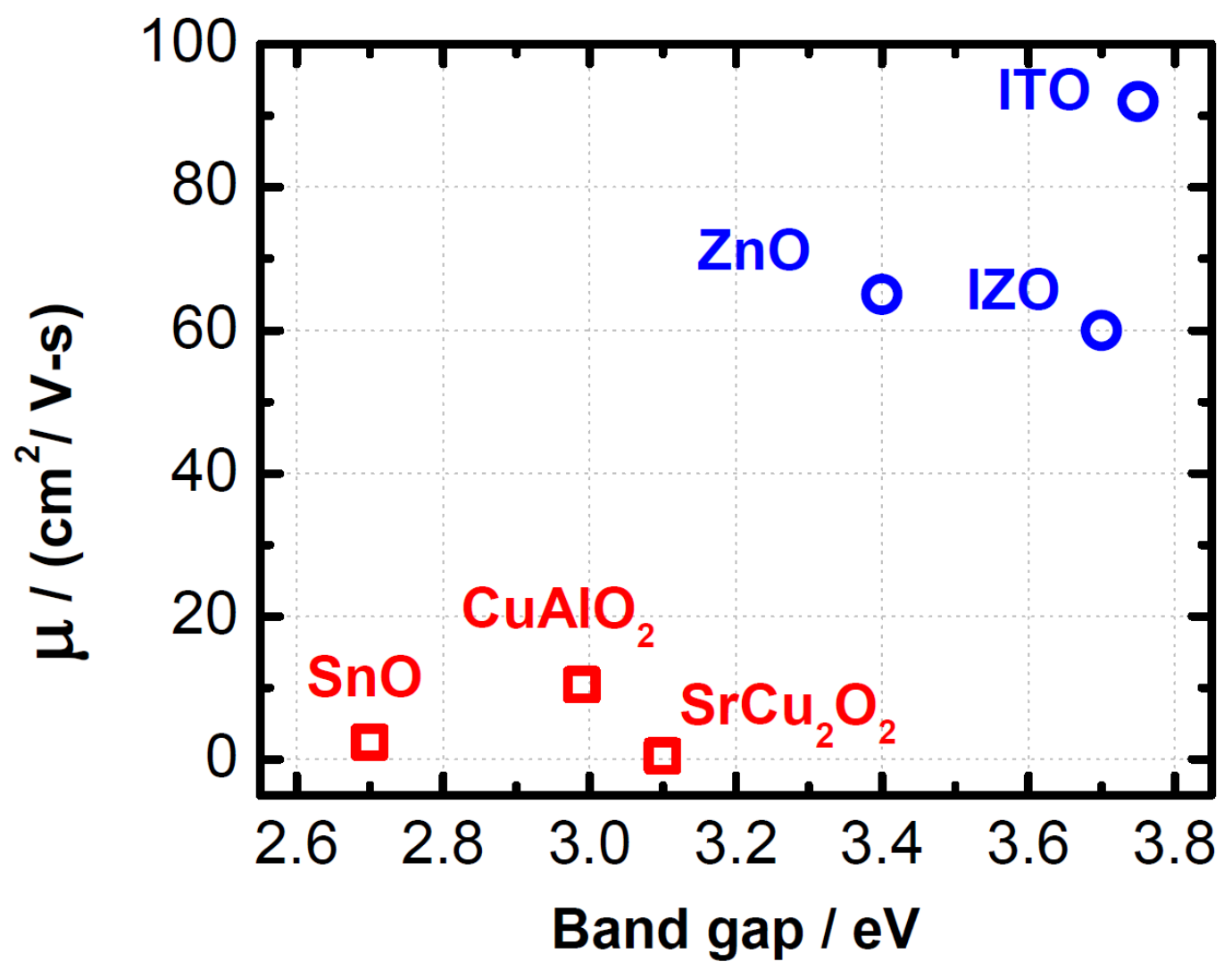

Figure 1.1 Illustrates a comparison of majority charge carrier mobility (in $\mathrm{cm} 2 / \mathrm{V} . \mathrm{s}$ ) vs band gap (in $\mathrm{eV}$ ) of a few state-of-the-art p-type (red) and n-type (blue) transparent conducting oxides. 


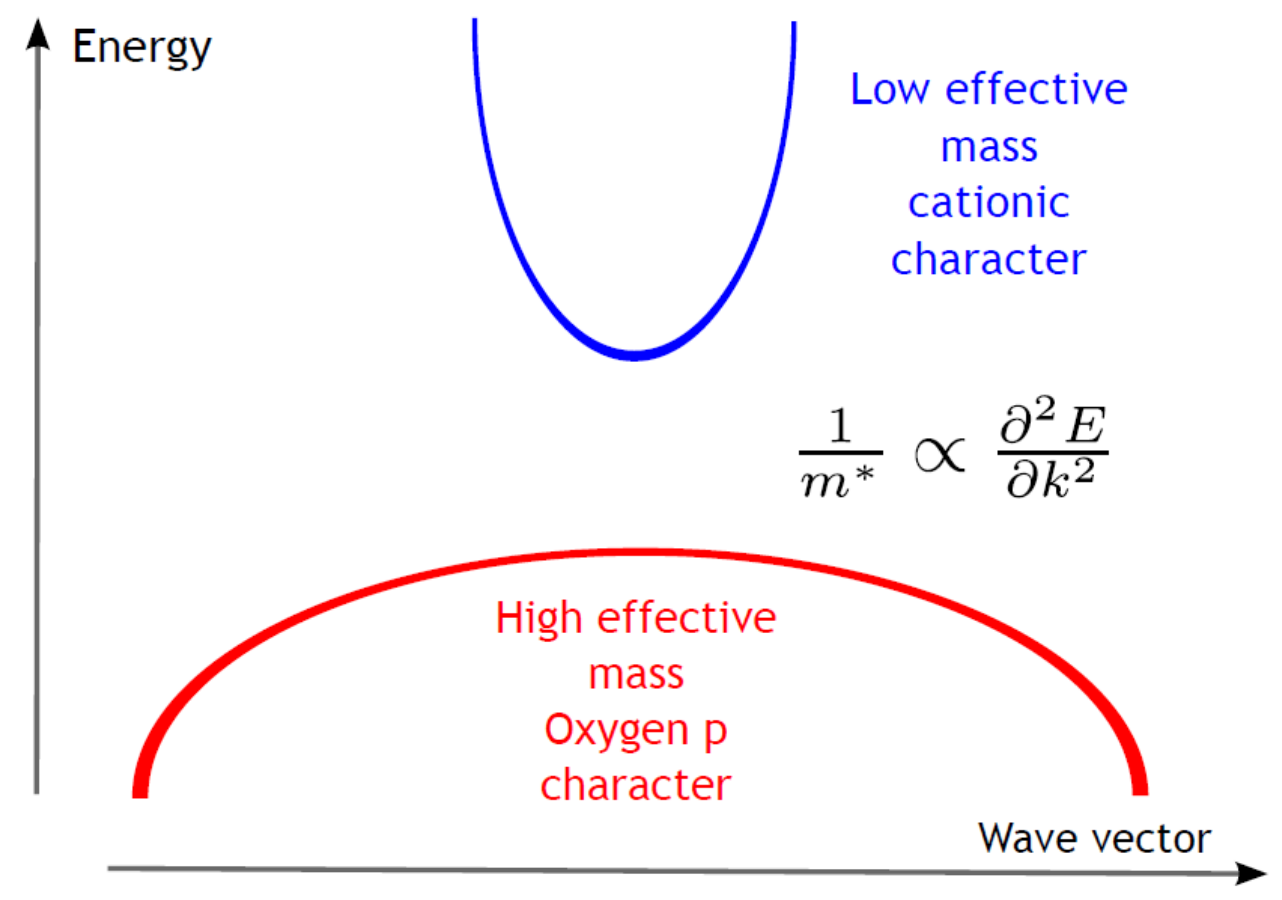

Figure 1.2 Illustrates the relationship between band curvature and charge carrier effective mass (and hence, mobility).

Illustrated in the Figure 1.2 is the relationship between band curvature and effective mass of the charge carriers (and hence charge carrier mobility). Majority of the oxides have flat valence bands (shown in red), resulting in high hole effective mass (and hence low hole mobility). This is majorly attributed to the presence of highly localized oxygen $2 p$ orbitals responsible for making the top of valence bands in majority of oxides ${ }^{25}$. Therefore, driven by the same realization Kawazoe et al., postulated that the key to unlocking high mobility is to delocalize the traditionally localized oxygen $2 p$ state, effectively decreasing the hole effective mass i.e. increasing the second derivative of the dispersion (shown in blue). They proposed an approach to delocalize the oxygen $2 p$ by introduction of highly electronegative metal cation that has energy levels similar to the oxygen $2 p$ to increase the extent of metal-oxygen covalency ${ }^{26}$. This led to the discovery of Cu-based p-type 
transparent conducting materials such as $\mathrm{CuAlO}_{2}, \mathrm{SrCu}_{2} \mathrm{O}_{2}$ etc ${ }^{21,23,25,27}$. These materials have been clearly found to show p-type conductivity, but the doping level and mobility are still low, typically in the range of $\mathrm{N} \leq 10^{18} \mathrm{~cm}^{-3}$ and $\mu \leq 10 \mathrm{~cm}^{2} / \mathrm{V}$.s.

Numerous research efforts have also been focused on doping $\mathrm{ZnO}$ p-type. Even though p-type conductivity has been shown to exist in $\mathrm{ZnO}$ by appropriate doping, it lacks stability and reproducibility considerably ${ }^{28}$. Search for a champion p-type material has not been limited to oxides. Several group around the world have worked on Hosono's hypothesis and have showed ptype conductivity in sulfides, selenides, oxysufides such as $\mathrm{LaCuOS}, \mathrm{BaCuSF}$ etc. Spinels of rare earth metals in the form $\mathrm{ZnM}_{2} \mathrm{O}_{4}(\mathrm{M}=\mathrm{Ir}, \mathrm{Rh}, \mathrm{Co})$ have also been reported.

In an effort to find p-type oxide with high mobility and transparency, researcher have also investigated the more spatially extended s-orbital chemistry. Introduction of metal cation with vacant s-orbital having energy similar to $\mathrm{O} 2 p$ has been postulated and demonstrated computationally to be more sophisticated in delocalizing the valence band as compared to the d-p hybridization as in the case of $\mathrm{Cu}$ based oxides ${ }^{7,29-31}$. However, so far the experimental realization of s-orbital chemistry oxides has been limited. Tin oxide, $\mathrm{SnO}\left(\mathrm{Sn}^{2+}\right.$ : $\left.[\mathrm{Kr}] 4 d^{10} 5 s^{2}\right)$ has been shown to be the best p-type s-orbital candidate, however with moderate hole mobility $\left(<5 \mathrm{~cm}^{2} / \mathrm{V} . \mathrm{s}\right)$, anisotropic transport, less stability, and moderate average transmission in the visible region (75$80 \%)^{7,8,30-32}$.

Clearly, the field of p-type transparent conducting oxides is still wide open and requires a breakthrough oxide material with antagonistic properties of wide band gap along with high hole mobility. $\mathrm{Bi}^{3+}\left([\mathrm{Xe}] 4 f^{14} 5 d^{10} 6 s^{2}\right)$ compounds provide creative solution for delocalizing the valence band by means of effective hybridization between completely filled $\mathrm{Bi} 6 s$ and $\mathrm{O} 2 p$ orbitals. However, the $\mathrm{Bi} 6 s$ states in current $\mathrm{Bi}$ compounds lie lower in energy as compared to $\mathrm{O} 2 p$. In 
order to achieve the maximum utilization of the s-p hybridization for modulating the hole effective mass, it is essential to find a perfect match of a suitable chemistry and structure that can support and promote the $\mathrm{Bi} 6 s-\mathrm{O} 2 p$ hybridization, while retaining the transparency.

Hautier et al. recently reported the use of high band gap and valence band curvature as a parameter for high-throughput computational screening to identify several highly promising compounds displaying exceptionally low effective masses (up to an order of magnitude lower than state-ofthe-art p-type TCOs) and wide band gaps. In this thesis, we have discussed the experimental realization of one such Bismuth based perovskite oxide, which has previously never been considered for the application of a transparent conductor. Further in order to study the structural, optical and electronic structure of the candidate material, various characterization techniques like X-ray diffraction, UV-Vis Spectroscopy, four point probe and hall measurements etc. were done. In the next few chapters we have presented the experimental synthesis, film deposition, structural, optical and electrical characterization of our candidate material, and used it to explain the high transparency and electronic band structure. We believe a strong interlinking and coordination between theory and experimentation is required to discover the novel set of materials. Our

concerted experimental efforts based on theory prediction points to the use of growing utility of data-driven material discovery and the combination of both informatics and experiment as an approach to discover future technologies.

\subsection{Scope of thesis}

Chapter 2 is devoted to the introduction of our candidate material, its computationally predicted band structure, electronic and optical properties, need for a suitable dopant. This chapter will also discuss in detail the methods used for synthesizing the single pure phase powder further used for 
depositing the films, utilized for optical and electrical characterization purposes. We will also mention the methods used for characterizing the samples.

Chapter 3 focuses on the discussion about characterization results of our candidate material. We will use these results to explain the band structure and make a comparison between the computationally predicted results and experimental observations. We will provide the basis for the discussion of s-p hybridization used for delocalization of valence band as a means to find low effective mass (and hence high mobility) p-type transparent conducting oxides.

Chapter 4 provides an outlook on the future work in the area of p-type transparent conducting oxides with particular attention paid to the importance of integrated theory-experiment efforts used for the realization of novel materials that have previously never been considered for the transparent conductor applications.

\section{Chapter 2 - Bismuth based p-type Perovskite Oxide}

\subsection{Identification of our candidate: $\mathrm{Ba}_{2} \mathrm{BiTaO}_{6}$}

In a bid to look for out of the box candidates to be used as high mobility p-type conductive oxides, researchers in the past have tried investigating the more spatially extended s-orbital chemistry. However, the experimental realization has been limited to $\mathrm{SnO}$ oxide, which has its own issues of stability in air along with average transmission and moderate mobility. $\mathrm{Bi}^{3+}\left([\mathrm{Xe}] 4 f^{14} 5 d^{10} 6 s^{2}\right)$ compounds offer another such alternative path for the utilization of the s-orbital based chemistry. However, as mentioned earlier the completely filled $s$-orbital of $\mathrm{Bi}^{3+}$ lies below the $\mathrm{O}-2 p$ in the valence band. Therefore, it is required to find a special chemistry and structure, which allows the 
most effective hybridization of $\mathrm{Bi}^{3+} s$-orbitals with the $\mathrm{O}-2 p$, thereby allowing the maximum delocalization of valence band.

Hautier et al. conducted a high-throughput computational search on thousands of binary and ternary oxides, and used high band gap and low hole effective mass as a means to identify the potential novel p-type transparent conducting oxides. Our candidate: $\mathrm{Ba}_{2} \mathrm{BiTaO}_{6}(\mathrm{BBT})$ was identified as a result of that high-throughput computational screening. While identifying this candidate three constraints were taken into consideration namely, low valence band effective mass in all the directions $\left(<0.5 \mathrm{me}_{\mathrm{e}}{ }^{*}\right)$, visible transparency (band gap $>3 \mathrm{eV}$ ) and high valence band maximum with respect to the vacuum (hence favoring p-type dopability) ${ }^{19}$.

\subsection{Our Candidate: $\mathrm{Ba}_{2} \mathrm{BiTaO}_{6}$}

\subsubsection{Structure}

BBT has a rhombohedral double perovskite structure $\left(\mathrm{A}_{2} \mathrm{BB}^{\prime} \mathrm{O}_{6}\right)$ as shown in the Figure 2.1, where A-site is occupied by the $\mathrm{Ba}^{2+}$, $\mathrm{B}$-site is occupied by $\mathrm{Bi}^{3+}, \mathrm{B}^{\prime}$-site by $\mathrm{Ta}^{5+}$ and $\mathrm{O}$ represents oxygen $^{33-35}$. 


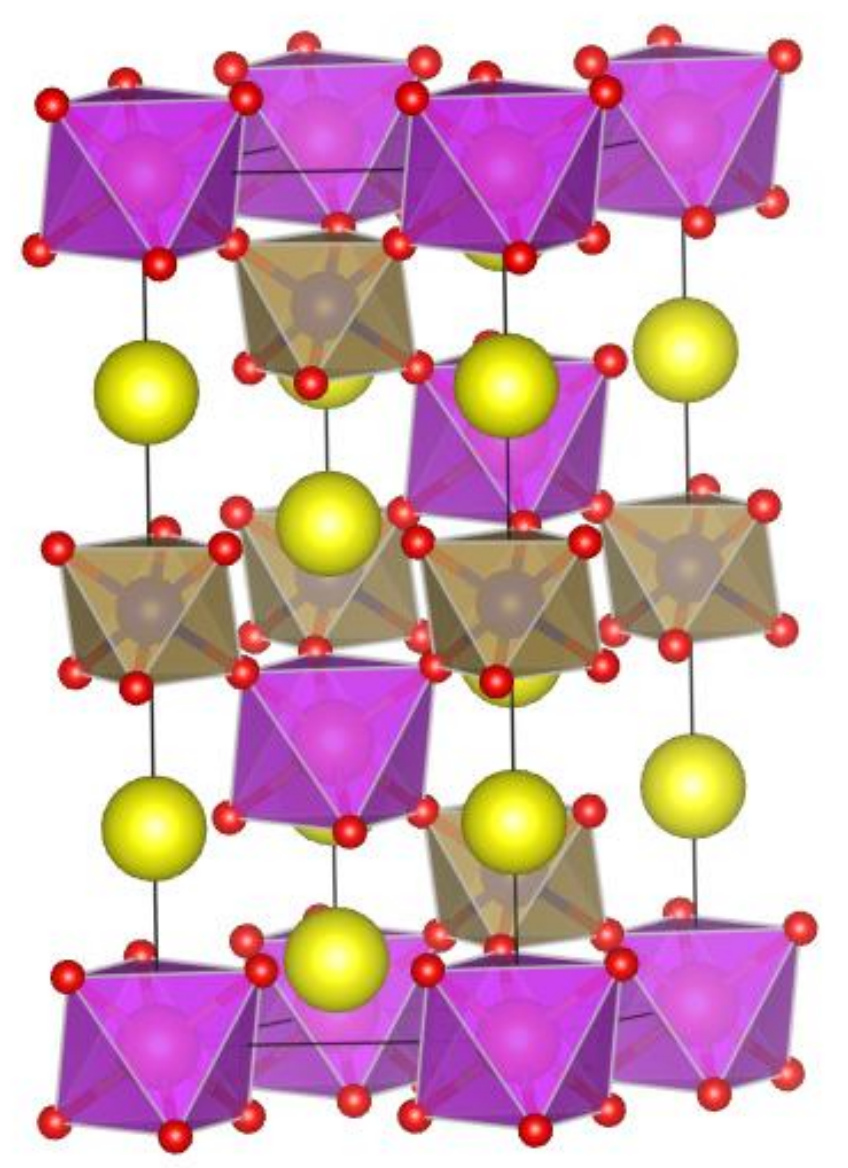

Figure 2.1 Structure of BBT (a double perovskite oxide of the form $\mathrm{A}_{2} \mathrm{BB}^{\prime} \mathrm{O}_{6}$ ), where yellow atoms represent $\mathrm{Ba}^{2+}(\mathrm{A}$-site), and red atoms represent oxygen, and atoms at the center of purple and grey octahedra represent $\mathrm{Bi}^{3+}(\mathrm{B}$-site $)$ and $\mathrm{Ta}^{5+}\left(\mathrm{B}^{\prime}\right.$-site) respectively.

Figure 2.1 shows the structure of $\mathrm{BBT} . \mathrm{Bi}^{3+}$ and $\mathrm{Ta}^{5+}$ are octahedrally coordinated to the oxygen atoms as shown in figure with purple and grey octahedra respectively.

\subsubsection{Band structure}


The unique qualities of BBT are 1. The low effective mass of its valence band, 2. Its high valence band maximum, which favors high hole mobility, and 3. Its large band gap, which gives the transparency in the visible range.

Figure 2.2 shows the computationally calculated GGA band structure of the rhombohedral BBT. A scissor operator was applied to the band gap to make it fit the single shot GW value. The calculated GW band gap for BBT was predicted to be $3.8 \mathrm{eV}$, well above the visible region ( 3.1 $\mathrm{eV})$. Along with the computationally predicted band structure of BBT, Figure 2.2 also illustrates the density of states projected on the different atomic sited using the GGA.
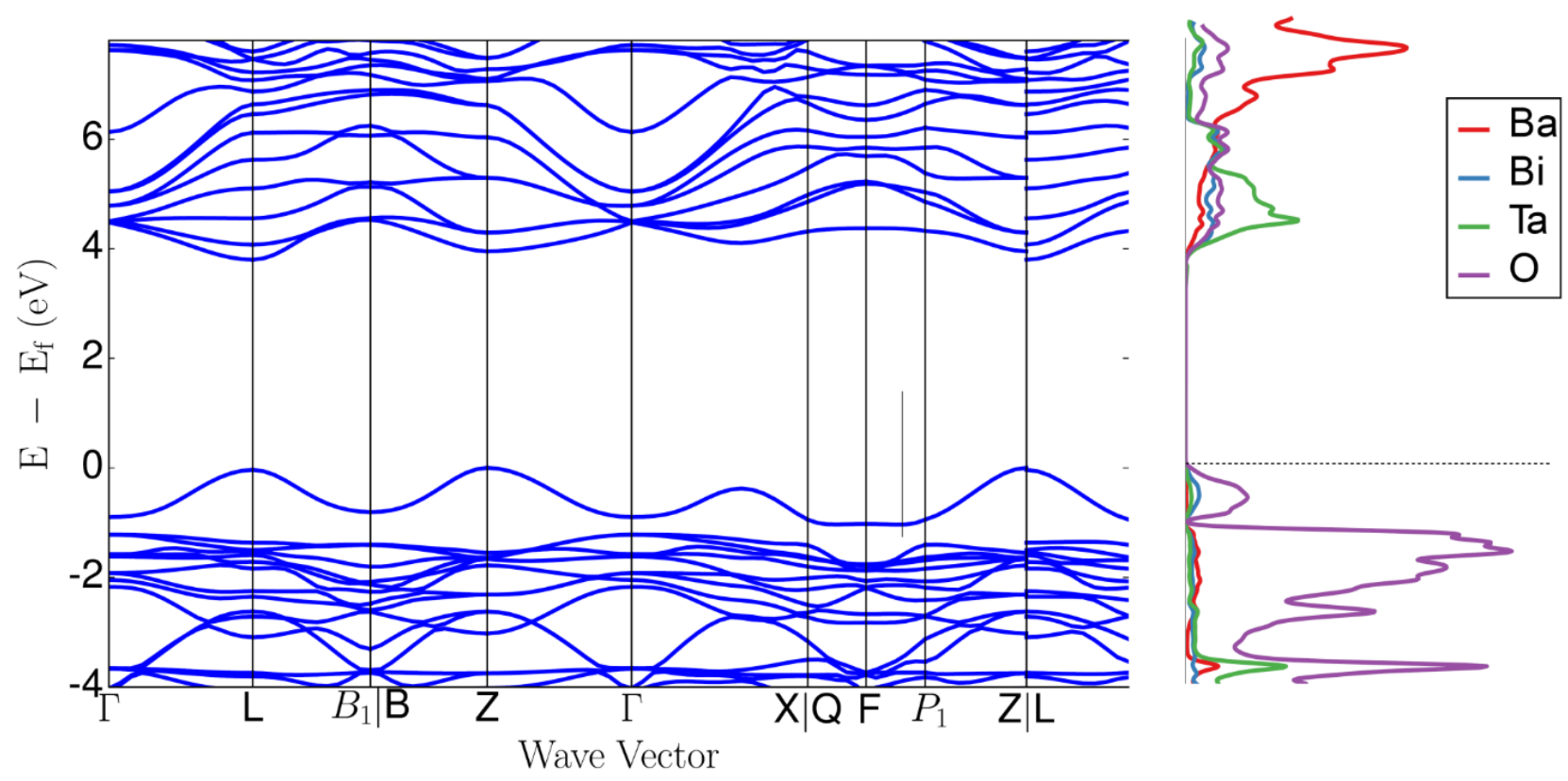

Figure 2.2 Illustrates the Band structure of $\mathrm{Ba}_{2} \mathrm{BiTaO}_{6}$ along symmetry lines and density of state projected on different atomic sites using GGA. A scissor operator was applied to the band gap to make it fit the single-shot GW value. 


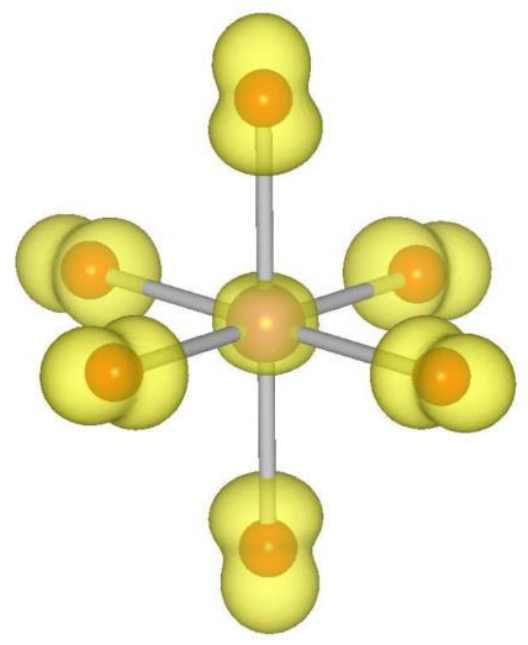

Figure 2.3 Illustrates square of the wave function in DFT-GGA for the valence band in $\mathrm{BiO}_{6}$ octahedra.

The first quality of low effective mass of the valence band in BBT stems from the mixed Bi 6s $\mathrm{O} 2 \mathrm{p}$ state as illustrated by the atomic projection of the valence band maximum (Fig. 2.2, bonding analysis is shown later). The charge density associated with the valence band shows that the Bi sorbital hybridizes with the oxygen p-orbital pointing toward the octahedron center while the two others perpendicular O p-orbitals stay non-bonding (Fig. 2.3). The conduction band on the other hand is of a mixed $\mathrm{Bi}$, Ta and $\mathrm{O}$ character and shows less dispersion (Fig. 2.1, 2.2). The dominant $\mathrm{Bi} 6 \mathrm{~s}-\mathrm{O} 2 \mathrm{p}$ interaction in the valence band in $\mathrm{BBT}$ is similar to the $\mathrm{BaBiO}_{3}$ perovskite, which has been extensively studied for its superconducting properties ${ }^{36-39}$. However, different from $\mathrm{BaBiO}_{3}$, which has an optical gap around $2 \mathrm{eV}^{40,41}, \mathrm{BBT}$ is transparent due to the presence of $\mathrm{Ta}^{5+}$ (instead of $\mathrm{Bi}^{5+}$ ), which pushes the conduction band upward and effectively increases the band gap. Interestingly, the electronic structure of BBT mimics what Sleight called the 'Holy Grail' electronic structure for p-type oxide with an s-based cationic state hybridizing with the oxygen's ${ }^{42}$. 
However, unlike other Bi-based oxides studied in the past, which generally have their Bi 6s state below the $\mathrm{O} 2 \mathrm{p}$, effectively limiting the $\mathrm{Bi} 6 \mathrm{~s}-\mathrm{O} 2 \mathrm{p}$ mixing, our chosen candidate has the $\mathrm{Bi} 6 \mathrm{~s}$ level close the $\mathrm{O} 2 \mathrm{p}$, which allows for a stronger $\mathrm{Bi} 6 \mathrm{~s}-\mathrm{O} 2 \mathrm{p}$ hybridization. At present, we attribute this unique Bi $6 \mathrm{~s}$ position to the BBT structure, although future study would be required to unravel the physical origin of this Bi 6s upshift.

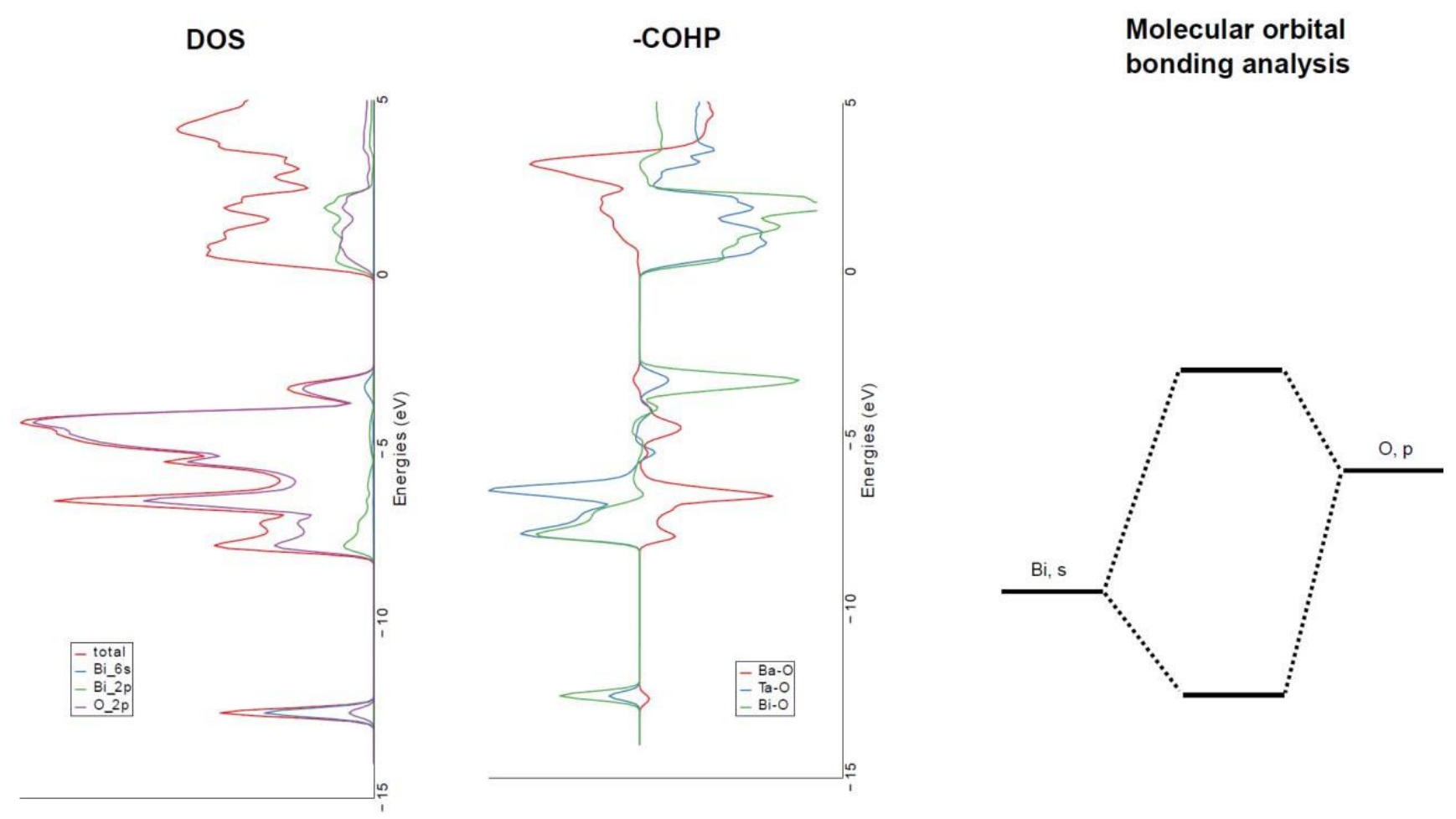

Figure 2.4 Illustrates density of state from a projection on localized basis sets using the lobster software on GGA-PBE results, (center) COHP analysis, and (right) schematic molecular orbital diagram for the $\mathrm{Bi} 6 s-\mathrm{O} 2 p$ hybridization. 
Hautier et al. performed an orbital overlap analysis in the COHP framework using the lobster software on their VASP GGA-PBE results ${ }^{19,43-47}$. Figure 2.4 shows that the valence band is formed by antibonding between $\mathrm{Bi} 6$ s and $\mathrm{O} 2 \mathrm{p}$ states.

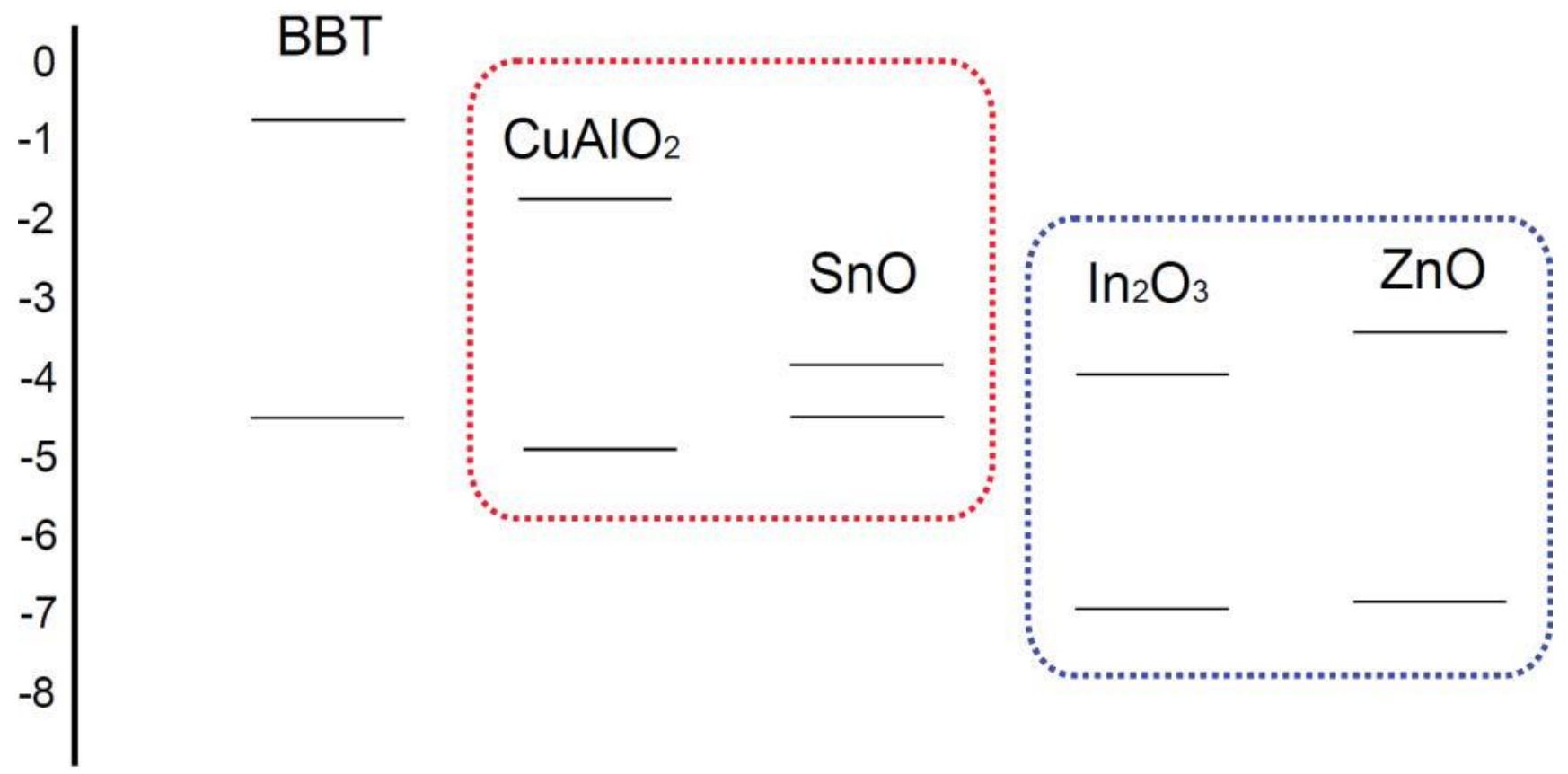

Figure 2.5 Illustrates the conduction band and valence band levels vs vacuum for BBT compared to typical n-type TCOs $\left(\mathrm{In}_{2} \mathrm{O}_{3}\right.$ and $\left.\mathrm{ZnO}\right)$ as well as p-type $\mathrm{TCOs}\left(\mathrm{CuAlO}_{2}\right.$ and $\left.\mathrm{SnO}\right)$.

The second important property of BBT is its p-type dopability, which is attributed to its high valence band. Figure 2.5 shows the conduction and valence bands vs vacuum of BBT and some state-of-the-art p-type $\left(\mathrm{SnO}, \mathrm{CuAlO}_{2}\right)$ and n-type $\left(\mathrm{In}_{2} \mathrm{O}_{3}, \mathrm{ZnO}\right)$ TCOs for comparison. In order to assess the typical nature of doping in BBT, Hautier et al. performed a slab computation on the (100) orientation of the rhombohedral BBT structure. The BBT slab computation was performed within GGA-PBE ${ }^{48-51}$ and they added the valence and conduction band correction from single- 
shot GW. Valence and conduction band edges can be used to assess dopability ${ }^{52}$. Comparing BBT's valence band edge to typical p-type and n-type TCOs, BBT is expected to behave more as a p-type than n-type material. Computations on a (110) slab have also given similar results.

\subsubsection{Effective mass}

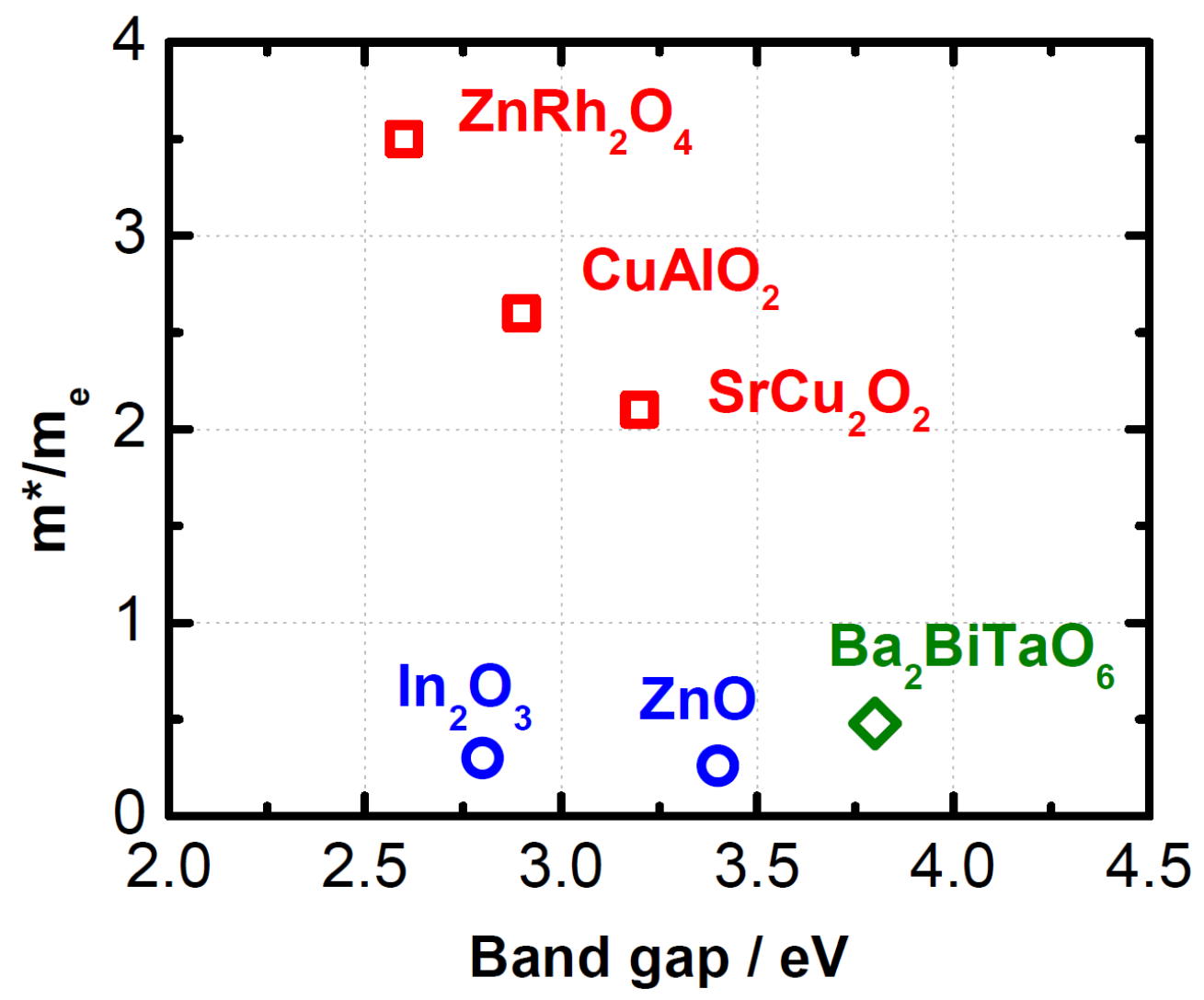

Figure 2.6 Illustrates the comparison of majority charge carrier effective mass and band gap of state-of-the-art p-type and n-type TCO with BBT (computationally predicted).

As mentioned earlier, the key to unlock high hole mobility or in other words low hole effective mass in $\mathrm{BBT}$ is attributed to its high $\mathrm{Bi} 6 s-\mathrm{O} 2 p$ hybridization, thereby delocalizing the valence 
band. Our collaborators, as a result of their computational efforts, predicted the band structure (as shown in previous figures), its shape and position, and made an estimation of hole effective mass in BBT. The hole effective mass of BBT was predicted to be $0.3 \mathrm{me}_{\mathrm{e}}{ }^{*}$, which is much lower than any of the existing p-type TCOs and in strong comparison with the champion n-type TCOs with majority charge carrier mobility $>100 \mathrm{~cm}^{2} / \mathrm{V}$-s. Figure 2.6 illustrates the comparison of effective 2mass of BBT with other state-of-the-art TCOs. The best candidate would lie in the bottom right corner of the figure, right where BBT is expected to be.

\subsection{Doping}

As we all know, doping is the process of introduction of impurities intentionally for the purpose of modulating the properties of the semiconductor. Briefly, dopants can be characterized as acceptor type or donor type depending on whether they result in extra holes or electrons in the system respectively. The quantity and position of dopant energy levels with respect to the intrinsic semiconductor's band structure decide the concentration of extra charge carriers produced. Usually, good dopant for a particular material are considered to be the ones that have energy levels within a few $\mathrm{kT}$ (where, $\mathrm{k}$ stands for Boltzman constant and $\mathrm{T}$ stands for temperature in Kelvin) of either the conduction band (for donor type impurity) or valence band (for acceptor type impurity) of the material.

Semiconductor materials can be doped intrinsically or extrinsically. A material is said to be doped intrinsically if the impurities are introduced unintentionally or intentionally during the synthesis of the material. 
In case of BBT (at a fixed set of chemical potentials: $\mu_{\mathrm{Bi}}, \mu_{\mathrm{Ba}}, \mu_{\mathrm{Ta}}$ and $\mu_{\mathrm{O}}$ ), we suspect that there may be some hole killing effects present in the system. These defects have a tendency to act as hole compensators or hole killers, thereby reducing the concentration of holes in the system. Therefore, BBT being a p-type semiconductor with holes as the majority charge carriers, it would be essential to find a perfect set of synthesis conditions that discourage the formation of aforementioned defects.

Due to the presence of notorious oxygen vacancies, without any extrinsic doping BBT exhibited negligible conductivity in both the pellet and thin film forms. We therefore examined the possibility of adding an electron acceptor to generate hole carriers. We elected to use $\mathrm{K}^{+}$for a $\mathrm{Ba}^{2+}$ substitution, as $\mathrm{K}^{+}$has a similar ionic size as $\mathrm{Ba}^{2+}$. We noted that $\mathrm{K}^{+}$was also previously used to substitute for $\mathrm{Ba}$ in $\mathrm{BaBiO}_{3}$ for superconductivity measurements. Our XRD (discussed in the next chapter) has revealed that $\mathrm{K}^{+}$can be substituted up to $35 \%$ of $\mathrm{Ba}^{2+}$ in $\mathrm{BBT}$ (forming $\left.\mathrm{Ba}_{1.3} \mathrm{~K}_{0.7} \mathrm{BiTaO}_{6}, \mathrm{BKBT}\right)$ with no noticeable secondary phase.

\subsection{Oxide synthesis}

In order to experimentally verify the properties and try to discover a new champion p-type TCO, we used solid synthesis methods to synthesize the pure phase powder of $\mathrm{BBT}$. $\mathrm{Ba}_{2-\mathrm{x}} \mathrm{K}_{\mathrm{x}} \mathrm{BiTaO}_{6}$ (with $\mathrm{x}$ ranging from 0 to 0.7 ) was prepared using a solid state synthesis method.

\subsubsection{Phase Diagram}


Before coming up with a perfect recipe for the synthesis of phase pure BBT, a few methods were tried and tested. Figure 2.7 shows the phase diagram of $\mathrm{BaO}, \mathrm{Bi}_{2} \mathrm{O}_{3}$ and $\mathrm{Ta}_{2} \mathrm{O}_{5}$.

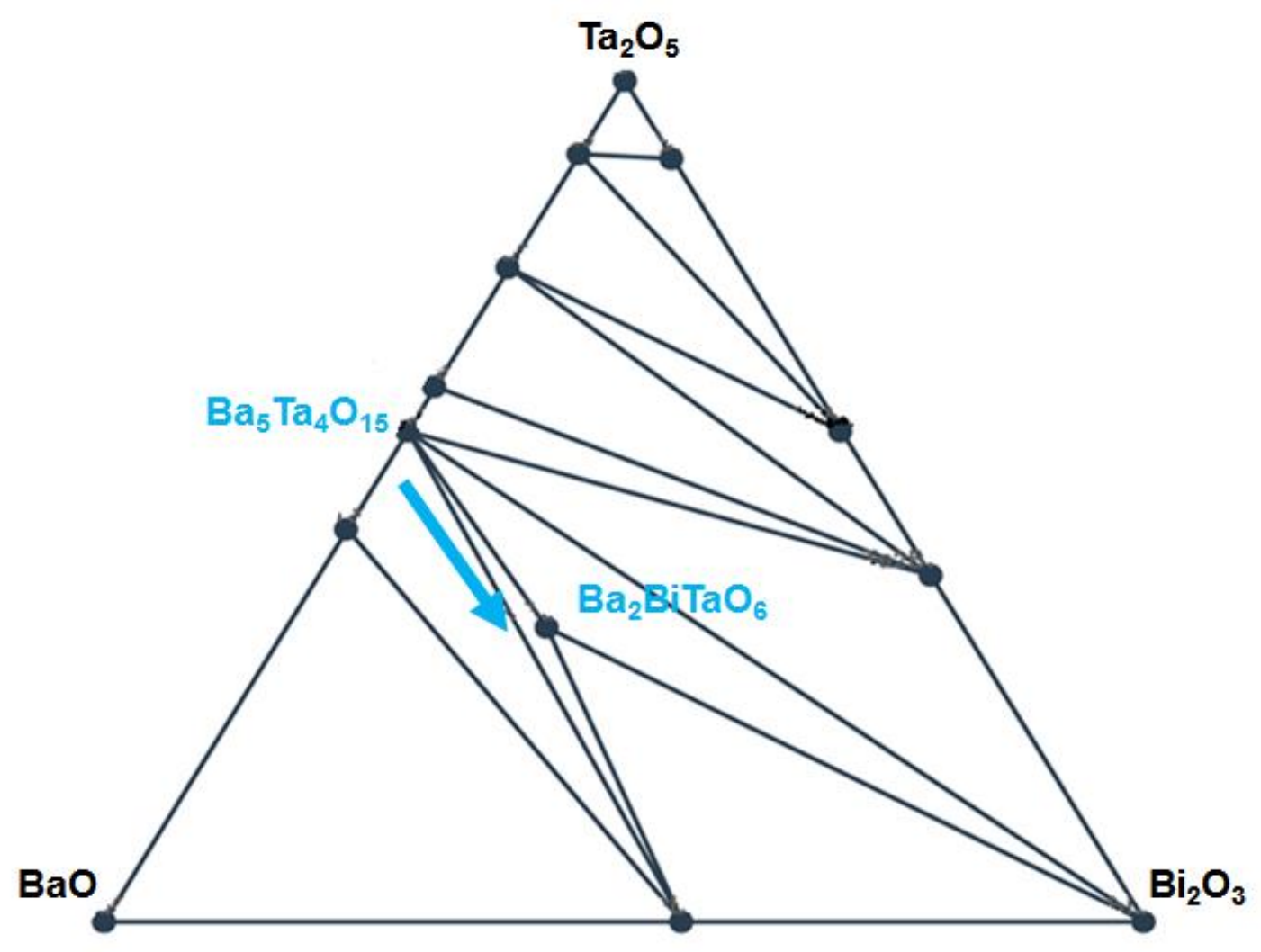

Figure 2.7 Illustrates the phase diagram of barium oxide $(\mathrm{BaO})$, bismuth oxide $\left(\mathrm{Bi}_{2} \mathrm{O}_{3}\right)$ and tantalum oxide $\left(\mathrm{Ta}_{2} \mathrm{O}_{5}\right)$ utilized for synthesizing the pure phase chemistry. Picture adopted from materialsproject.org.

As shown in the phase diagram, $\mathrm{Ba}_{2} \mathrm{BiTaO}_{6}(\mathrm{BBT})$ lies right near the center in the phase diagram. This correct composition can only be achieved, if the right amount of starting mixture, along with the perfect synthesizing conditions like oxygen potential, temperature, environment etc. are maintained. 


\subsubsection{Solid state method}

The perfect recipe for synthesizing pure phase BBT is as following: Stoichiometric amounts of $\mathrm{BaCO}_{3}\left(99.95 \%\right.$, Alfa Aesar), $\mathrm{Bi}_{2} \mathrm{O}_{3}\left(99.999 \%\right.$, Alfa Aesar) and $\mathrm{Ta}_{2} \mathrm{O}_{5}(99.993 \%$, Alfa Aesar) were thoroughly ground with agate mortar and pestle. Then, the mixture was pressed into a pellet using a hydraulic press (Grimco). The stoichiometric mixture thus pressed into a pellet was heat treated in a furnace at $1100{ }^{\circ} \mathrm{C}$ for 12 hours under flowing argon (Ultrahigh purity, Airgas) atmosphere. This method of synthesizing BBT was found to be the most effective way. A lot of other techniques and recipe were tried as well. The resultant products thus obtained was crushed, grinded and further analyzed using characterization techniques like X-ray diffraction, scanning electron microscopy, X-ray photoelectron spectroscopy etc.

\subsubsection{Film deposition}

Pulsed laser deposition (PLD, Neocera) was used to deposit thin films of $\mathrm{Ba}_{2-\mathrm{x}} \mathrm{K}_{\mathrm{x}} \mathrm{BiTaO}_{6}$ onto (100) $\mathrm{MgO}$ single-crystalline substrates (MTI). A pulsed laser beam from a $\mathrm{KrF}$ excimer laser $(\lambda$ $=248 \mathrm{~nm}$ ) was focused onto the target that was formed from the re-ground and re-pressed oxide powders. The fluence energy and repetition rate of the laser were adjusted to achieve a deposition rate of $\sim 5 \mathrm{~nm} / \mathrm{min}$. The substrate temperature and oxygen pressure were maintained at $650^{\circ} \mathrm{C}$ and $7.5 \times 10-4$ torr. After the deposition, the films were annealed at $400^{\circ} \mathrm{C}$ for 5 hours in an oxygen pressure of 1 torr. The deposition conditions for BKBT were decided by keeping into consideration the elimination of hole killers (oxygen vacancies and anit-site defects), which for BBT have high formation energies at high oxygen potential (high oxygen pressure and low temperatures). 


\subsection{Characterization}

\subsubsection{Structural: X-Ray Diffraction}

X-Ray diffraction (XRD) is one of the most important tools used for identifying the atomic structure of the materials. The diffractometer in an XRD machine collects and analyses the X-rays diffracted from the various crystallographic orientations of the sample. The technique is generally used for the identification of chemical phases present in the material. Apart from that, XRD is also utilized for the identification of various structural parameters like lattice parameters, crystal structure, inter-atomic spacing, grain size in crystalline or polycrystalline materials, epitaxy etc. Crystal structures of the powder samples and films were characterized by Scintag Theta-Theta Xray Diffractometer, Bruker General Area Detector Diffraction System, and Rigaku SmartLab Xray Diffractometer using $\mathrm{Cu} \operatorname{K} \alpha$ radiation $(\lambda=1.54052 \AA)$.

\subsubsection{Optical: UV-Visible Spectrometer}

$\mathrm{UV}-\mathrm{Visible} \mathrm{spectrometer} \mathrm{is} \mathrm{used} \mathrm{for} \mathrm{measuring} \mathrm{the} \mathrm{transparency,} \mathrm{absorbance} \mathrm{and} \mathrm{reflectance} \mathrm{data}$ of the specimen films. A beam of light of various wavelength is made to fall on the specimen and its response is recorded by the detector. The optical properties such as band gap (both direct and indirect), refractive index etc. can be estimated using the transparency, absorbance data from the spectrometer.

Estimating the transparency, band gap, absorption coefficient etc. optical properties of the BBT and BKBT films was one of the most important part of the thesis. Films deposited using the method mentioned previously were utilized for measuring the aforementioned properties using UV-VisNIR Spectrometer (Shimazdu). 


\subsubsection{Ellipsometer}

Ellipsomtery is an optical technique which is used for investigating the dielectric properties, refractive index, thickness and composition of the thin films. A polarized light is made incident on the specimen and change in polarization of the reflected light is measured. This change in polarization is used to get information about the refractive index and thickness of the films.

\subsubsection{Four-point probe Technique}

Four-point probe technique is one of the most common and straight forward technique used for measuring the resistivity or sheet resistance of a material (conducting or semi-conducting thin films) of a uniform thickness. The apparatus consists of four needles, by passing the current in outer two needles and measuring the resultant voltage in the inner two probes provides a measure of resistivity of the substrate. It is also used for measuring the doping concentration from the resistivity of the sample.

We utilized four-point probe technique as mentioned for measuring the resistivity and sheet resistance of our BKBT films deposited onto $\mathrm{MgO}$ substrates, which were further utilized for optimizing the synthesis and deposition conditions of the films by PLD to minimize the resistivity.

\subsubsection{Van der Pauw Technique}

Van der Pauw technique is utilized for measuring the sheet resistance/ resistivity of the conducting/semiconducting samples. Four-point probe technique cannot identify the polarity of 
the charge carriers. Van der Pauw technique utilizes four ohmic contacts made on the four corners of the film and electrical resistivity of the layer is then measured by multiplying the sheet resistance with the thickness of the layer.

Powder samples of BKBT were pressed into pellets of $\sim 1 \mathrm{~mm}$ thickness, which was then cut into square bar specimens $(\sim 7 \times 7 \mathrm{~mm})$ using diamond saw. The conductivity was measured using van der Pauw technique (Keithley) on contacts made from Ag-ink covered Ga-In eutectics (SigmaAldrich). Hole carrier density $\left(\mathrm{n}_{\mathrm{p}}\right)$ and Hall mobility $\left(\mu_{\mathrm{H}}\right)$ were determined using Physical Property Measurement System (PPMS, Quantum Design) with Au/Ti electrodes at room temperature. 


\section{Chapter 3 - Results and Discussion}

To experimentally verify the prediction, we synthesized a phase-pure BBT using a solid state synthesis method as mentioned in chapter 2 . The phase purity was verified with X-ray diffraction studies (XRD, Figure 3.1). We separated the experimental characterization into two parts. The first part focused on the optical characterization of BBT. To evaluate the optical band gap, we conducted a transmission measurement on a BBT thin film using the BBT powder as a pulsed laser deposition (PLD) target for the thin film deposition. Using a single-crystal (100) $\mathrm{MgO}$ as a substrate for growth, we obtained a textured BBT film with film thickness estimated to be $\sim 120$ $\mathrm{nm}$ using spectroscopic ellipsometry. The optical transmittance of the BBT film had an average value of $>90 \%$ in the wavelength range between the $350-800 \mathrm{~nm}$. The direct optical band was estimated to be $>4.5 \mathrm{eV}$. The difference with the GW computed band gap $(3.8 \mathrm{eV})$ could come from the difference between the optical and electronic gap, or from the possible gap underestimation present in the single-shot GW.

\subsection{X-Ray Characterization}

Phase pure powder of BBT was synthesized by the solid state method as described in the previous chapter. X-ray diffraction studies were done on the samples synthesized in the furnace. All the samples were synthesized at $1100 \mathrm{C}$ for $12 \mathrm{hrs}$ in Argon atmosphere. 


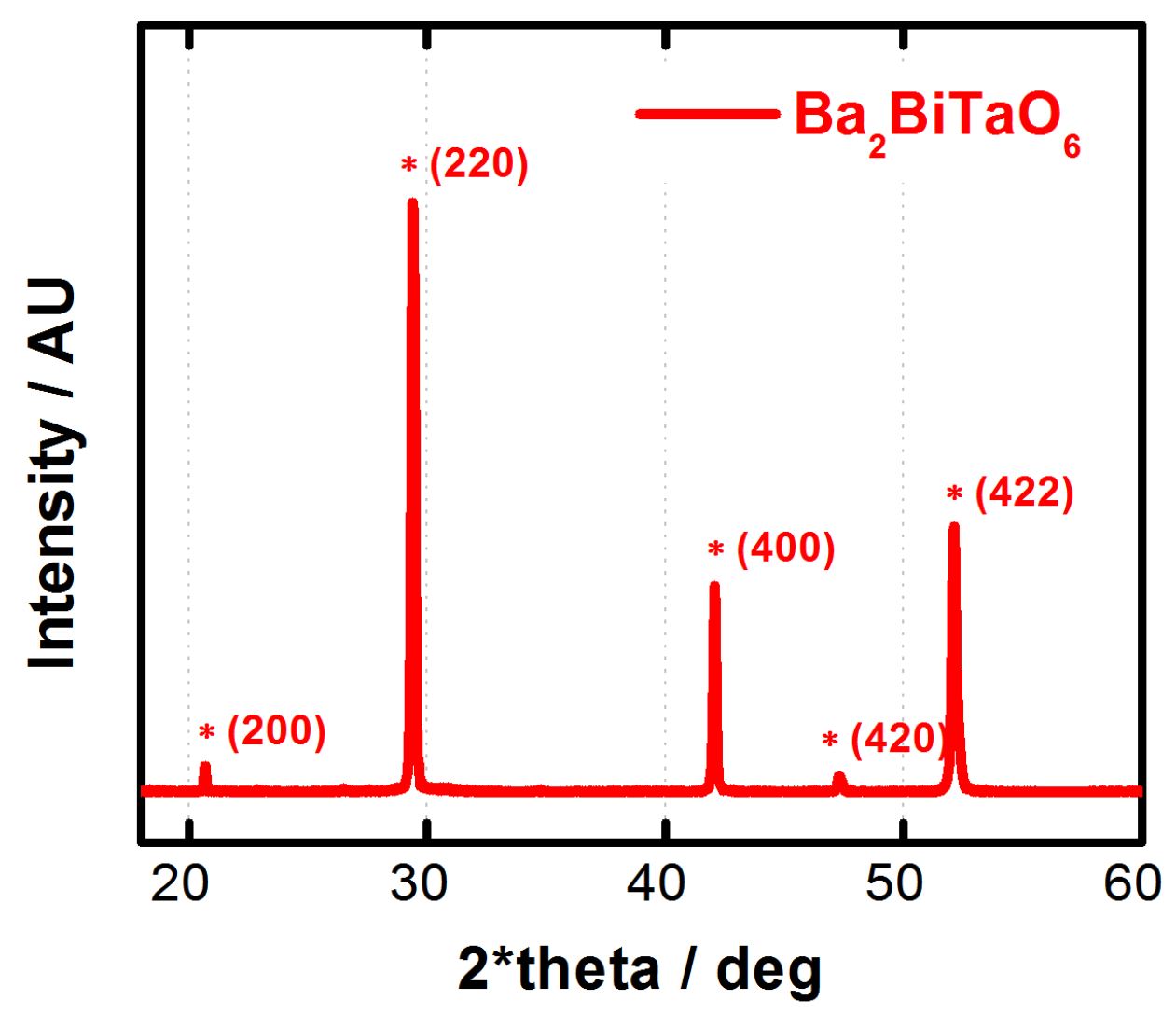

Figure 3.1 Illustrates the phase pure X-ray diffraction data of BBT synthesized at 1100C, $12 \mathrm{hrs}$ in argon atmosphere.

As shown in the figure 3.1 all the peaks were matched with the ICSD database and no secondary impurity phases were detected. The structure is found to be cubic ( $\mathrm{Fm} \overline{3} \mathrm{~m}$ space group) with a lattice constant to be around $0.842 \mathrm{~nm}$.

Without extrinsic doping, BBT exhibited negligible conductivity in both the pellet and the thin film form. We therefore examined the possibility of adding an electron acceptor to generate hole carriers. We elect to use $\mathrm{K}^{+}$for a $\mathrm{Ba}^{2+}$ substitution as $\mathrm{K}^{+}$has a similar ionic size as $\mathrm{Ba}^{2+}$. We note that $\mathrm{K}^{+}$was also previously used to substitute for $\mathrm{Ba}$ in $\mathrm{BaBiO}_{3}$ for superconductivity ${ }^{36}$. Our XRD 
reveals that $\mathrm{K}^{+}$can be substituted up to $35 \%$ of $\mathrm{Ba}^{2+}$ in $\mathrm{BBT}$ (forming $\mathrm{Ba}_{1.3} \mathrm{~K}_{0.7} \mathrm{BiTaO}_{6}$, $\mathrm{BKBT}$ ) with no noticeable secondary phase (fig 3.2).

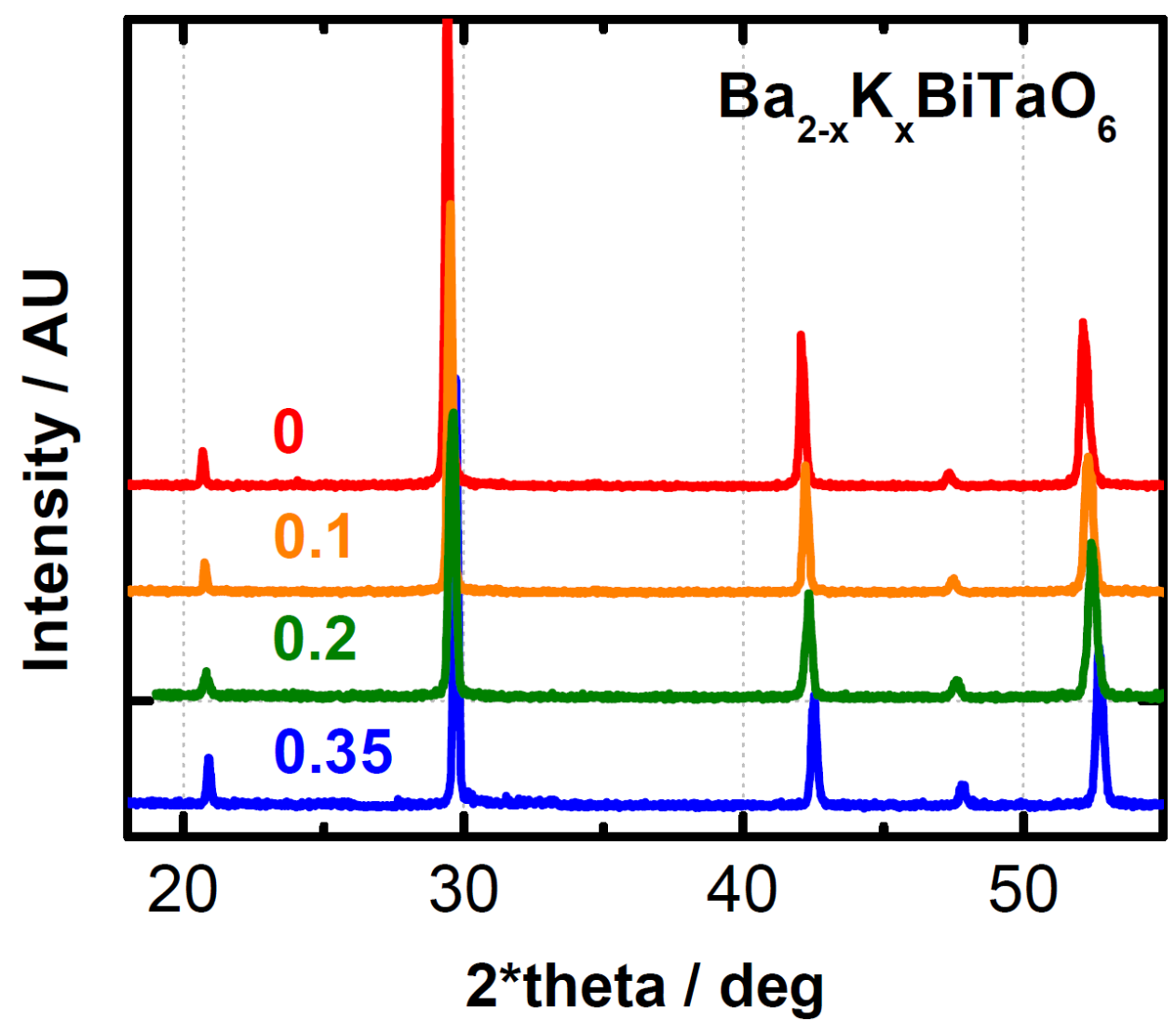

Figure 3.2 Illustrates the $\mathrm{X}$-Ray diffraction studies done on $\mathrm{Ba}_{2-\mathrm{x}} \mathrm{K}_{\mathrm{x}} \mathrm{BiTaO}_{6}(\mathrm{BKBT})$, where $\mathrm{x}=(0$ $-0.35)$. No extra secondary impurity phases were detected. 


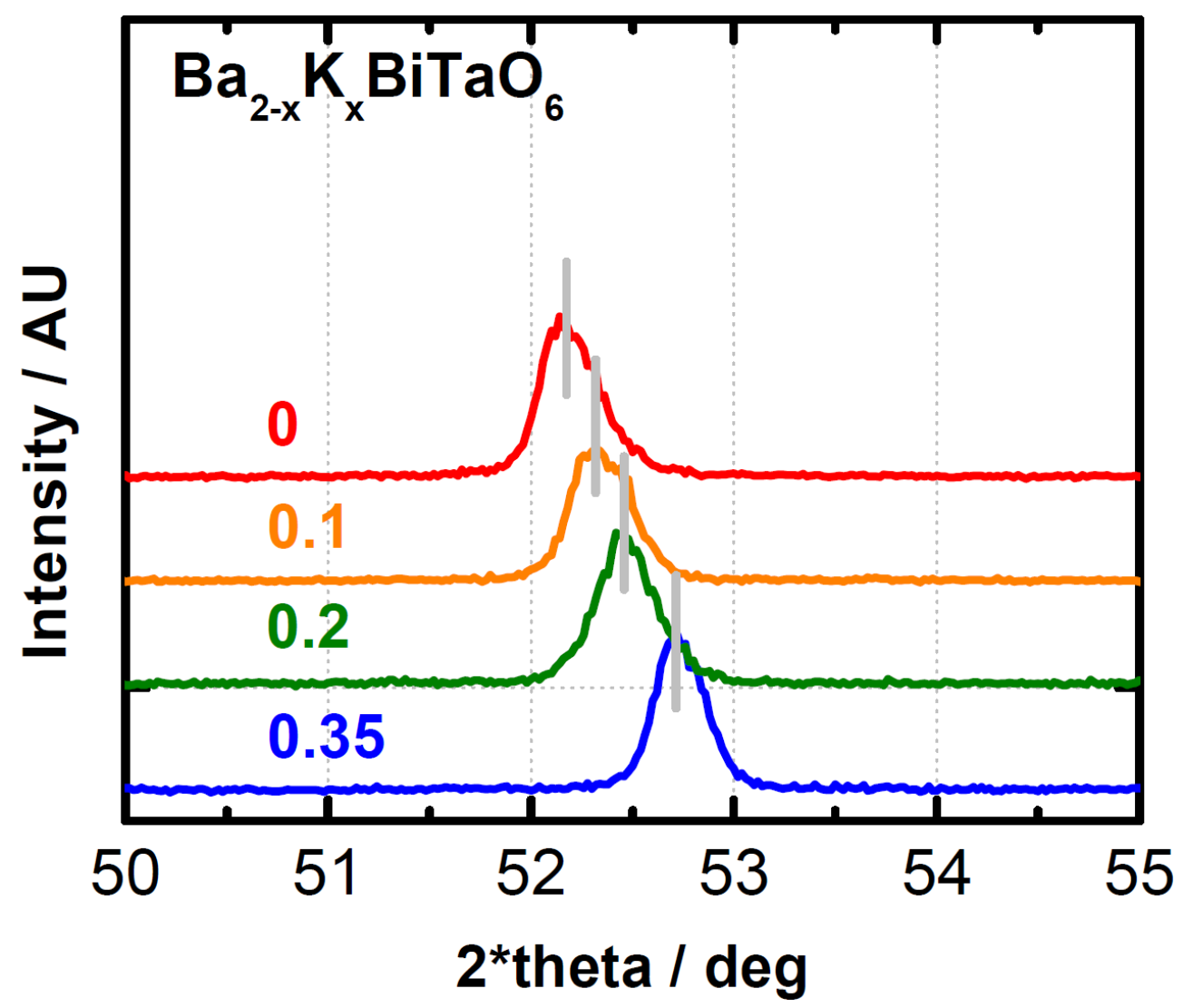

Figure 3.3 Illustrates the peak shifting as $\mathrm{K}$ is added as a substitutional impurity in place of $\mathrm{Ba}$ in BKBT.

As more amounts of potassium $(\mathrm{K})$ is introduced in the BKBT system, peak shifting toward the higher values of 2-Theta was observed, indicating a decrease in the interatomic spacing (d) and hence shrinkage of unit cell. 


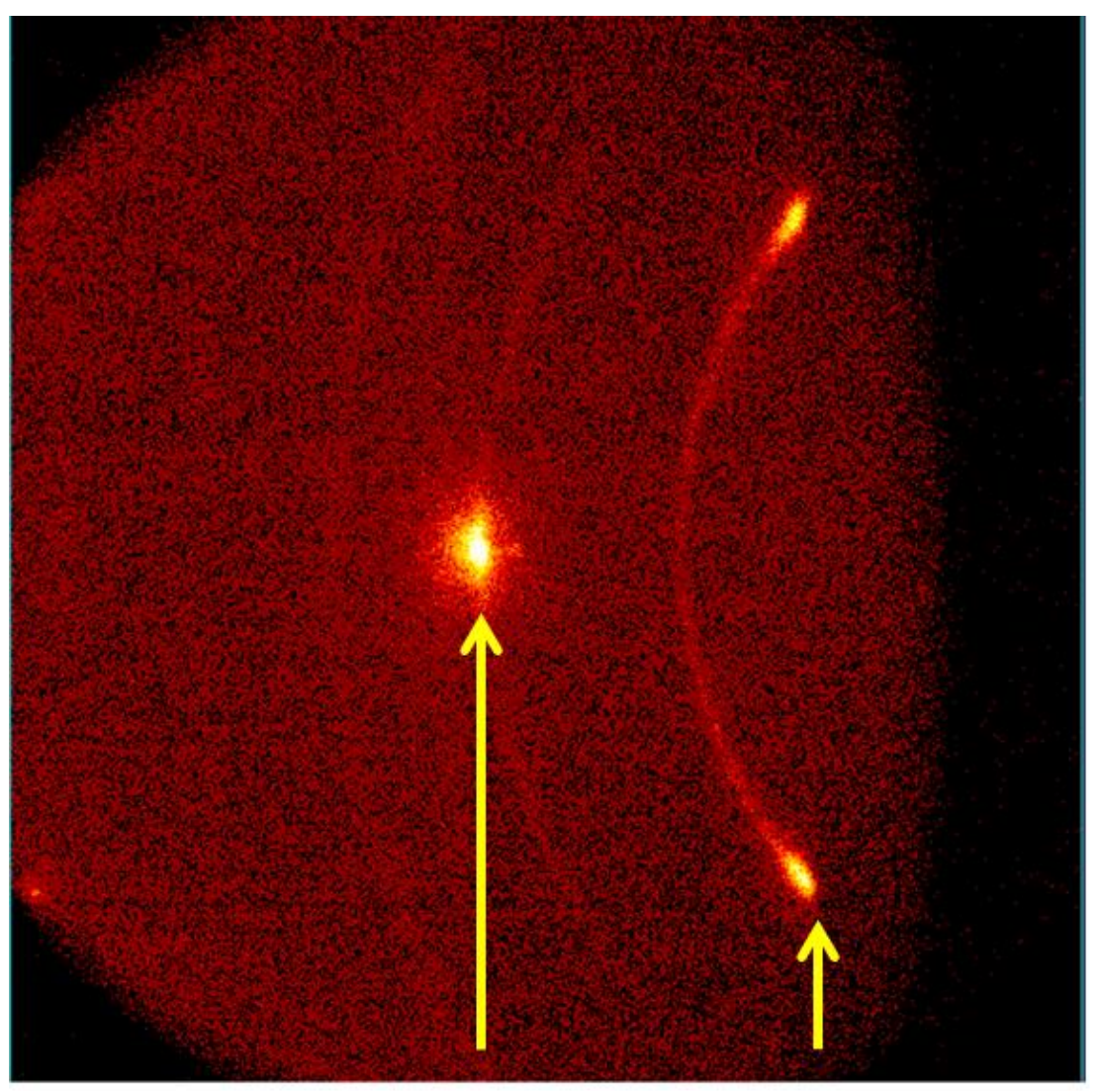

\section{$\mathrm{MgO}(400) \quad \mathrm{Ba}_{2} \mathrm{BiTaO}_{6}(220)$}

Figure 3.4 Bruker General Area Detector Diffraction System (GADDS) of $\mathrm{Ba}_{2} \mathrm{BiTaO}_{6}$ thin films.

As mentioned in the previous chapter, the phase pure powder synthesized was grinded and repressed by a hydraulic press into a cylindrical pellet. This pellet was then used as a target for depositing thin films of BBT onto (100) MgO using pulsed laser deposition technique. Figure 3.4 shows the Bruker General Area Detector Diffraction System (GADDS) of the BBT film deposited. The film deposited were textured in nature (not completely epitaxial) as can be seen from the 2Theta-phi scan shown in the figure 3.5 below. 


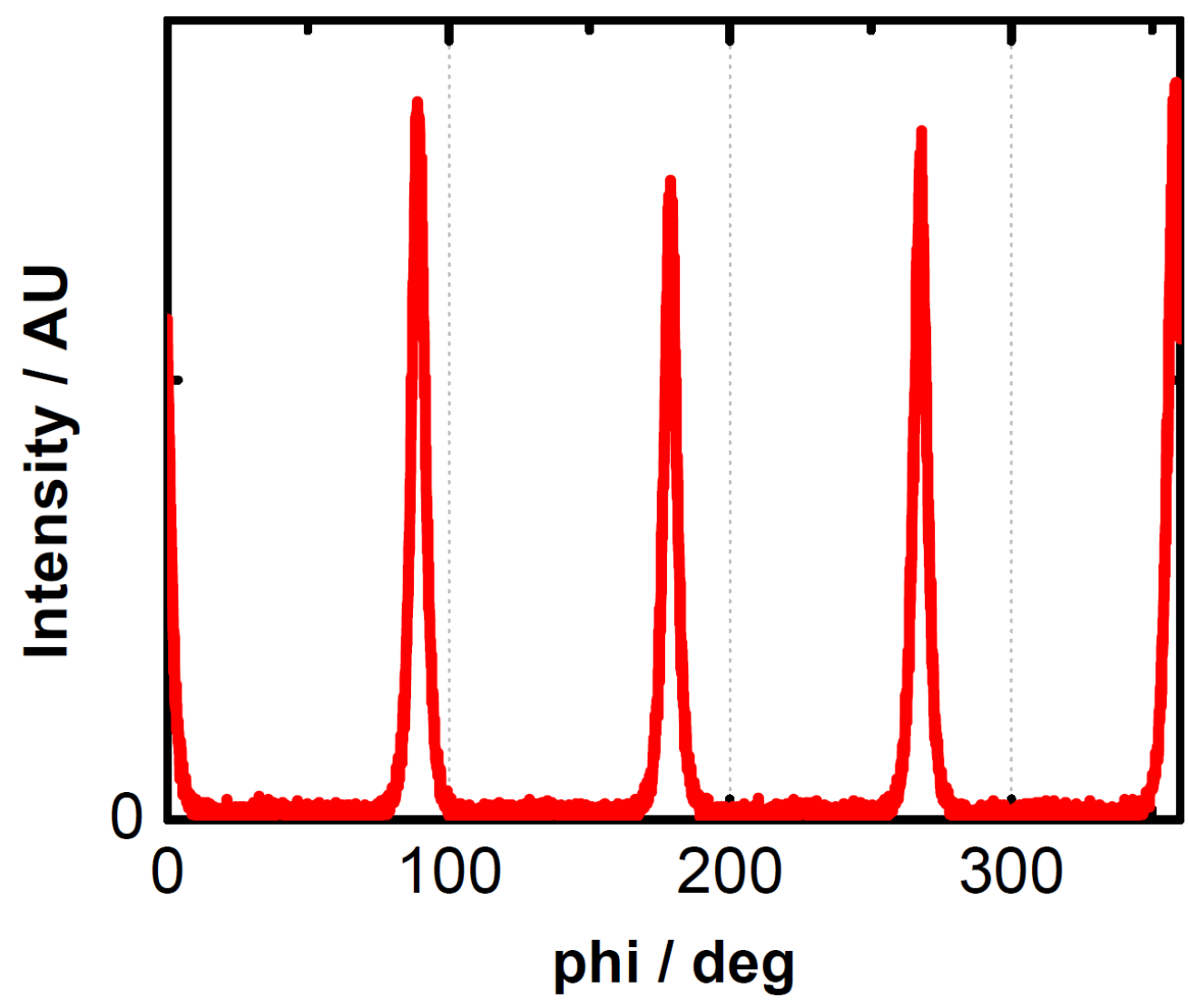

Figure 3.5 2Theta-phi scan done on the $\mathrm{Ba}_{2} \mathrm{BiTaO}_{6}$ films deposited onto (100) $\mathrm{MgO}$ substrate.

In a similar manner as mentioned, thin film of BKBT were deposited as well, in order to understand the effect of $\mathrm{K}$ addition in place of barium in BBT, whether it affects the transparency of the films. Figure 3.6 and 3.7 shows the 2Theta-Theta and 2Theta-phi scan done on the BKBT films, which were later utilized for the transparency and other optical measurements. 


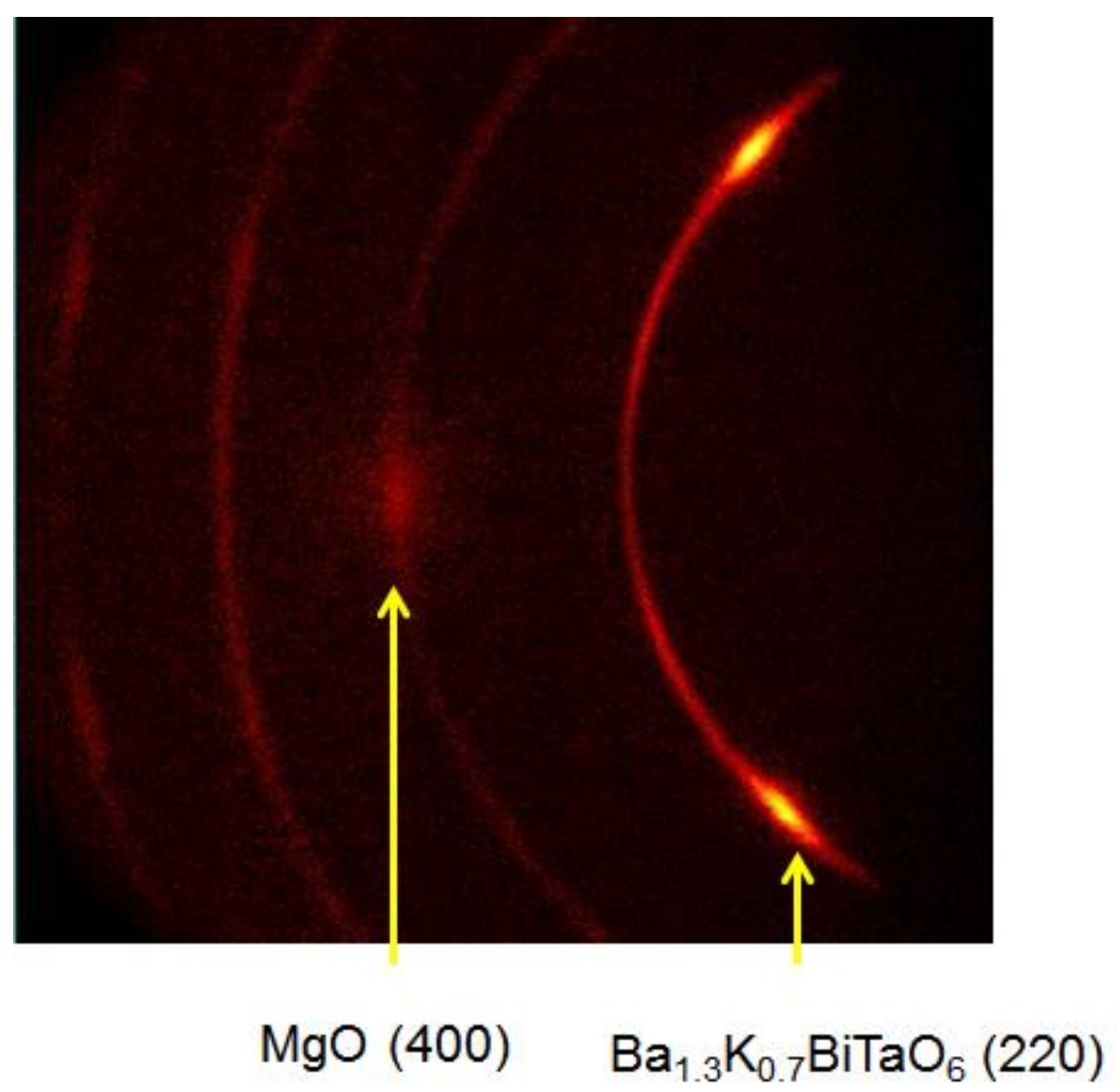

Figure 3.6 Bruker General Area Detector Diffraction System (GADDS) of $\mathrm{Ba}_{1.3} \mathrm{~K}_{0.7} \mathrm{BiTaO}_{6}$ thin films. 


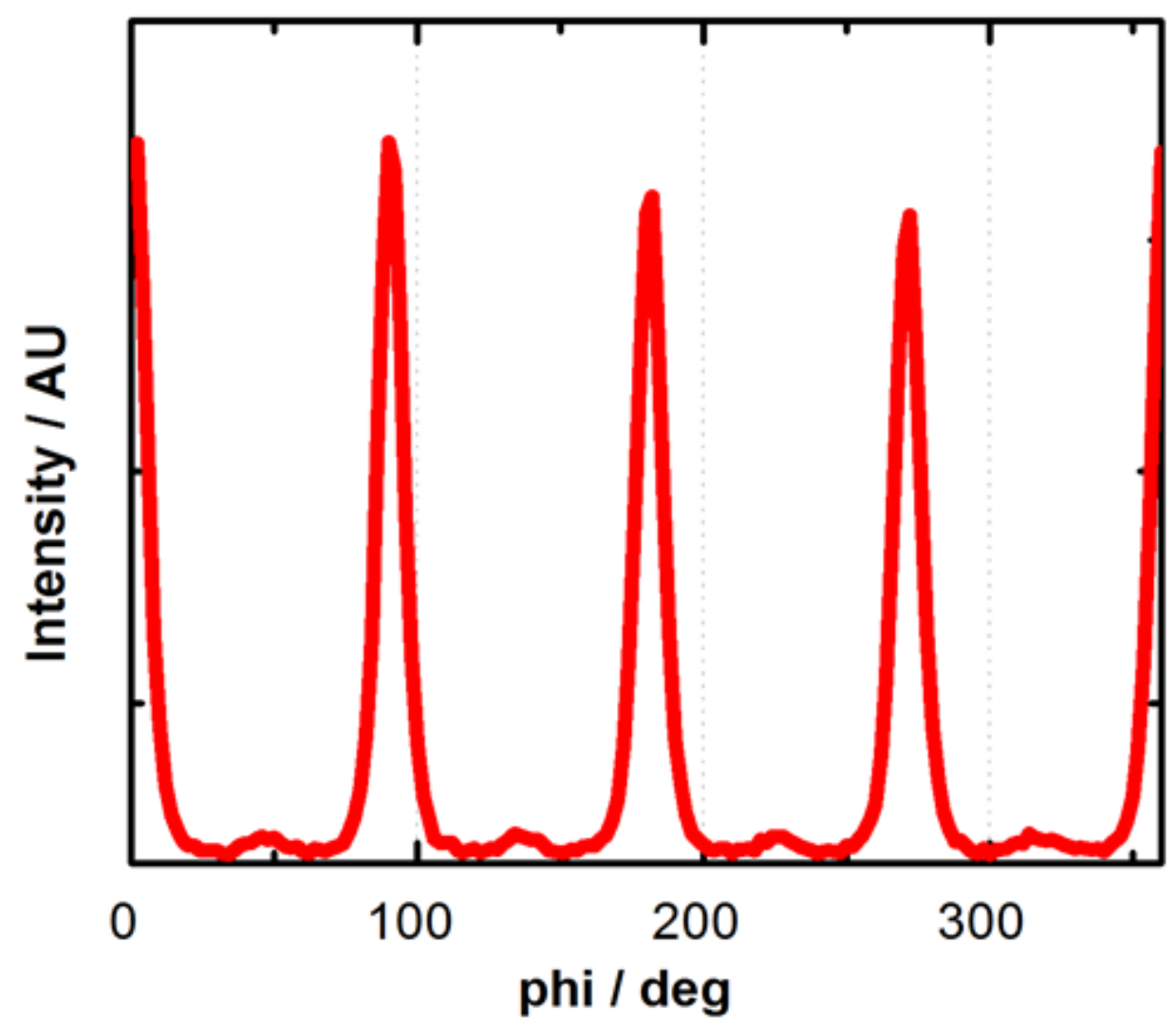

Figure 3.7 2Theta-phi scan done on the $\mathrm{Ba}_{1.3} \mathrm{~K}_{0.7} \mathrm{BiTaO}_{6}$ films deposited onto (100) $\mathrm{MgO}$ substrate.

\subsection{Optical measurements}

The $\mathrm{K}^{+}$substitution has small effects on the sample optical property; the PLD deposited BKBT film shows that the visible transparency was largely preserved (>90\%, Fig. 3.8). 


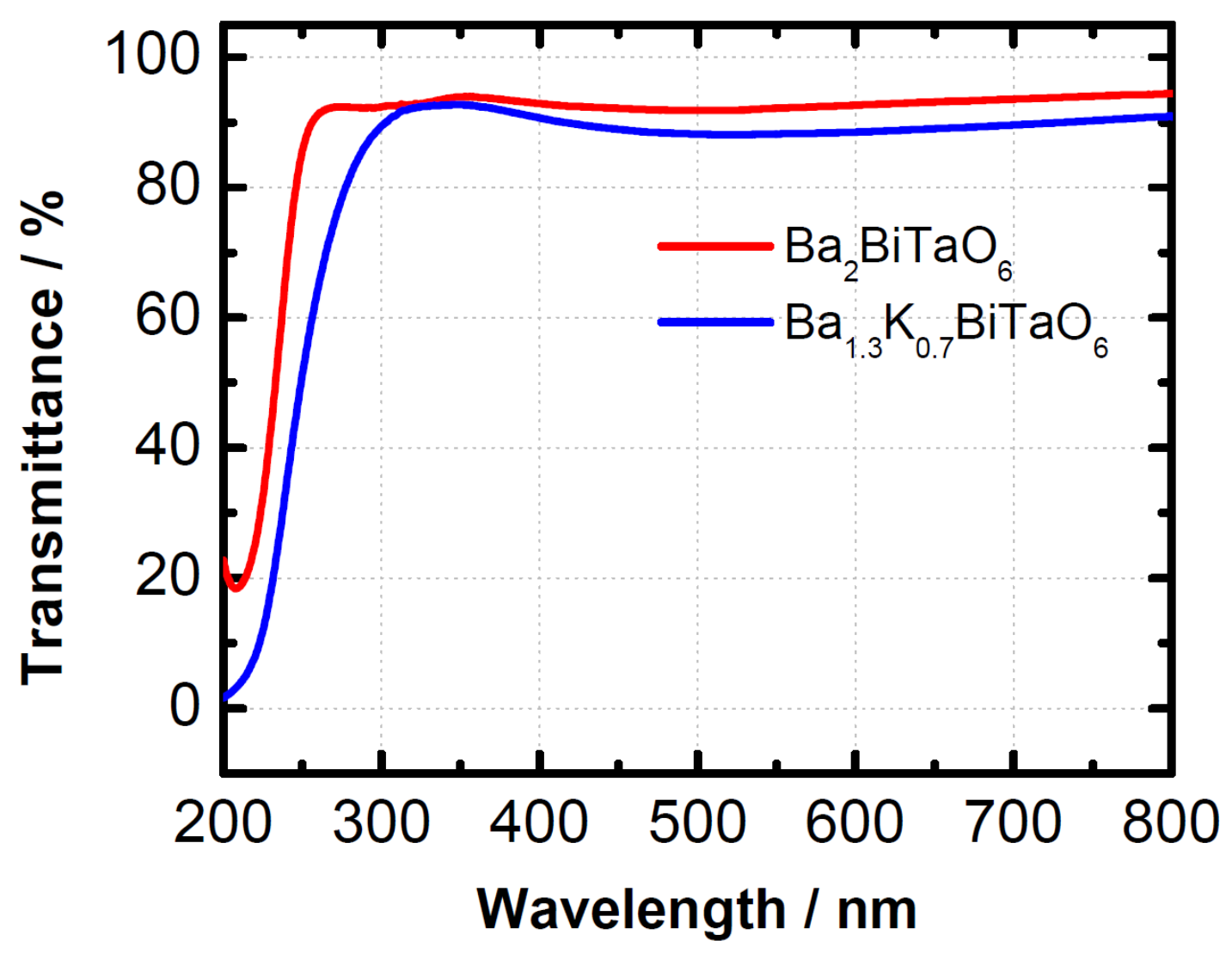

Figure 3.8 Transparency vs wavelength data from UV-Vis Spectrometer in wavelength range from $200-800 \mathrm{~nm}$ for BBT vs BKBT films. Transparency of the films was maintained well above $90 \%$ even after adding $\mathrm{K}=35 \%$ in $\mathrm{BKBT}$.

Based on this observation, we suspect that the $\mathrm{K}^{+}$inclusion did not lead to any optically active, mid-gap state formation. We point out that the optical band gap of our material decreases with $\mathrm{K}^{+}$ concentration (Fig. 3.9, 3.10). This optical gap change could be from the reduction in the lattice parameter once $\mathrm{K}^{+}$substitutes in the $\mathrm{Ba}^{2+}$ position. 


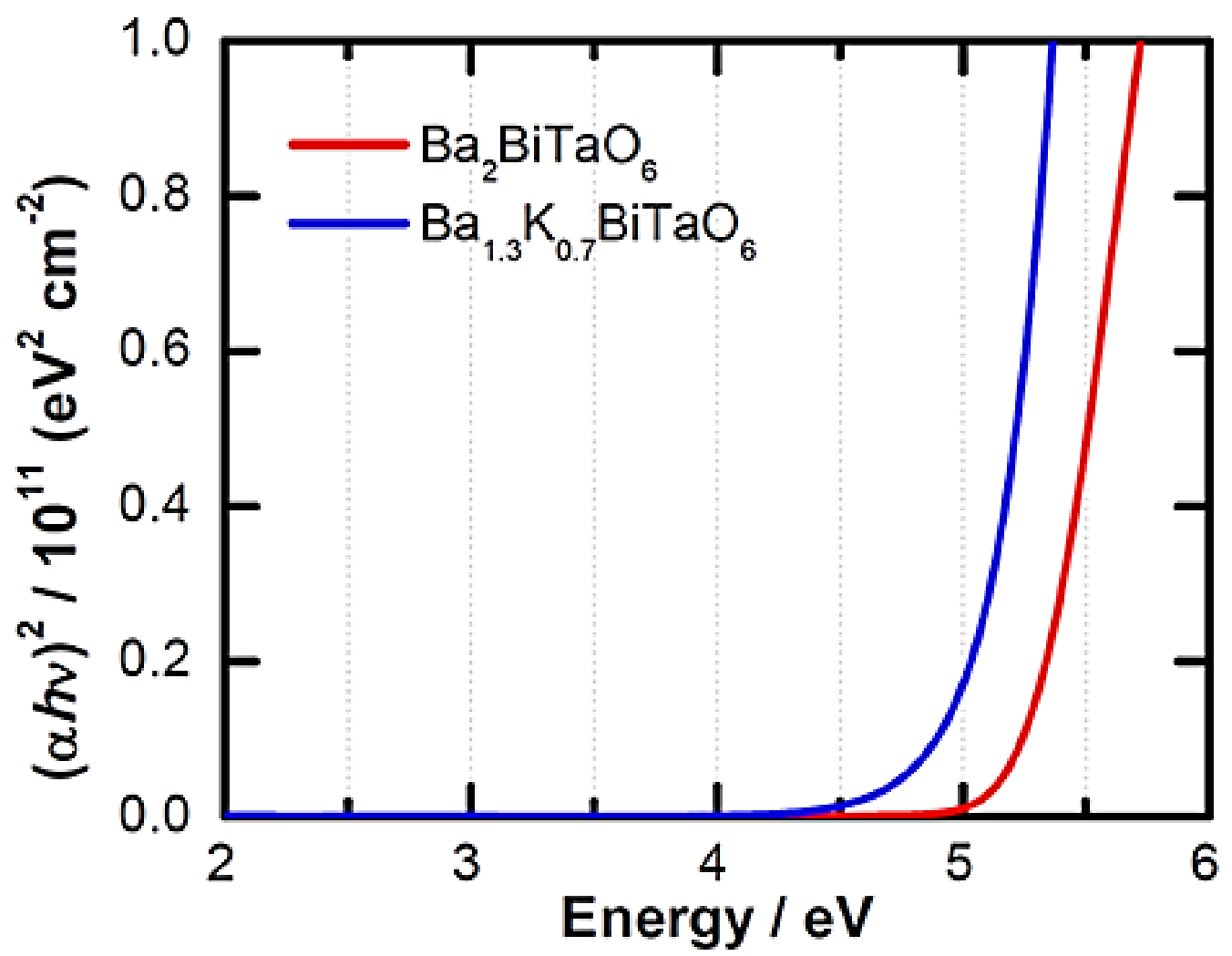

Figure 3.9 Illustrates the $(\alpha \mathrm{h} v)^{2}$ vs Energy plot of the $\mathrm{Ba}_{2-\mathrm{x}} \mathrm{K}_{\mathrm{x}} \mathrm{BiTaO}_{6}$ films grown on $\mathrm{MgO}$ (100) substrate. A rough estimate of direct band gap of the material can be made from this curve. 


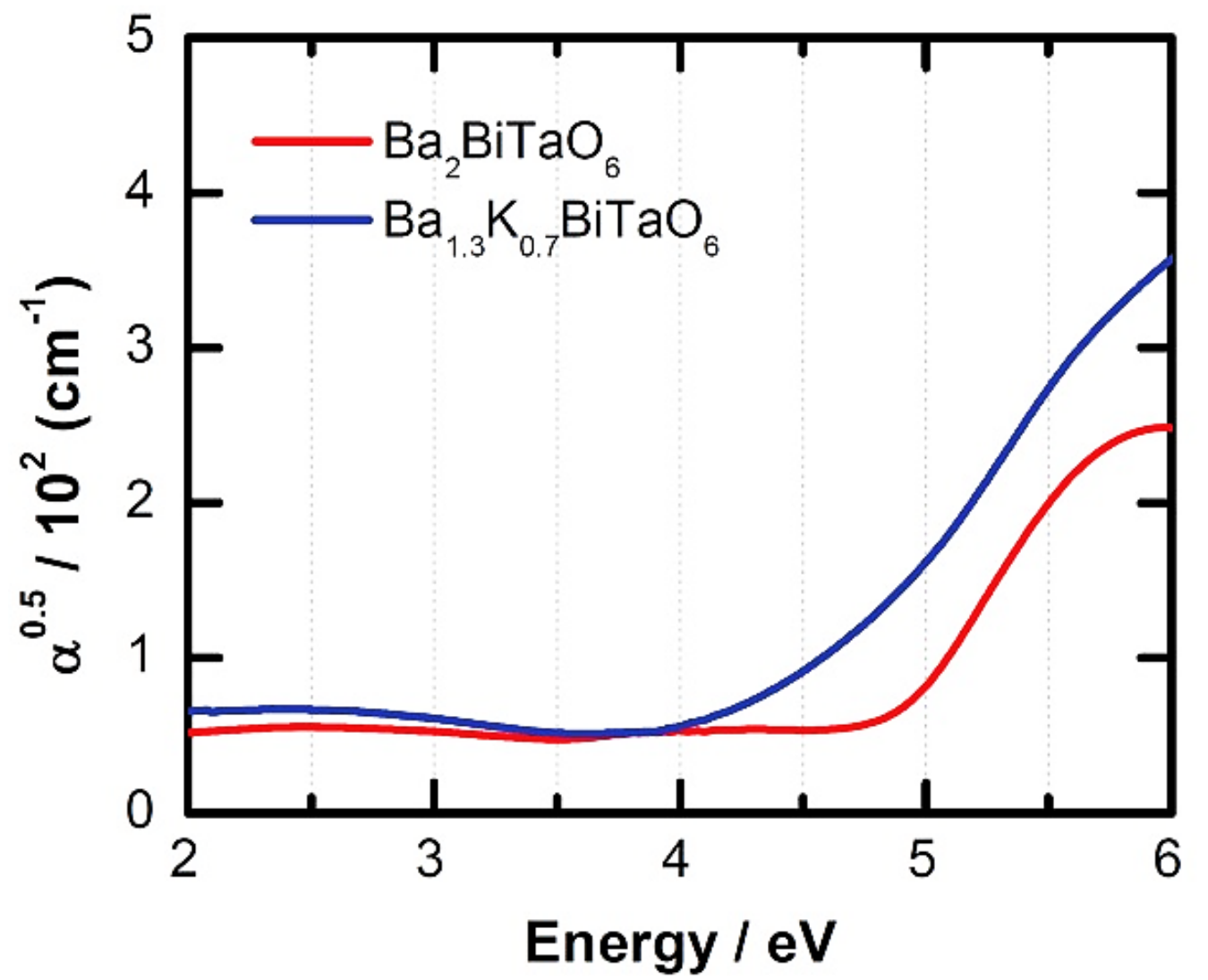

Figure 3.10 Illustrates the $(\alpha)^{0.5}$ vs Energy plot of $\mathrm{Ba}_{2-\mathrm{x}} \mathrm{K}_{\mathrm{x}} \mathrm{BiTaO}_{6}$ films grown on $\mathrm{MgO}$ (100) substrate. A rough estimate of indirect band gap of the material can be made from this curve.

\subsection{Hall measurements}

Having demonstrated the visible transparency of both BBT and BKBT, we now focus on evaluating the transport property of the materials. Without $\mathrm{K}$ incorporation, BBT exhibits no detectable conductivity, behaving as an intrinsic wide-band-gap semiconductor. We therefore focus on the BKBT sample, specifically $\mathrm{Ba}_{1.3} \mathrm{~K}_{0.7} \mathrm{BiTaO}_{6}$, for the transport measurements. Our four-point probe measurements on the BKBT pellets reveal the conductivity in the range of $\sim \mathrm{k} \Omega$ $\mathrm{cm}$. This level of conductivity precludes the possibility for a direct Hall measurement on the BKBT 
film. To obtain meaningful a Hall result, we instead focus on the BKBT pellet, whose geometry has a sufficient thickness to support the Hall experiment.

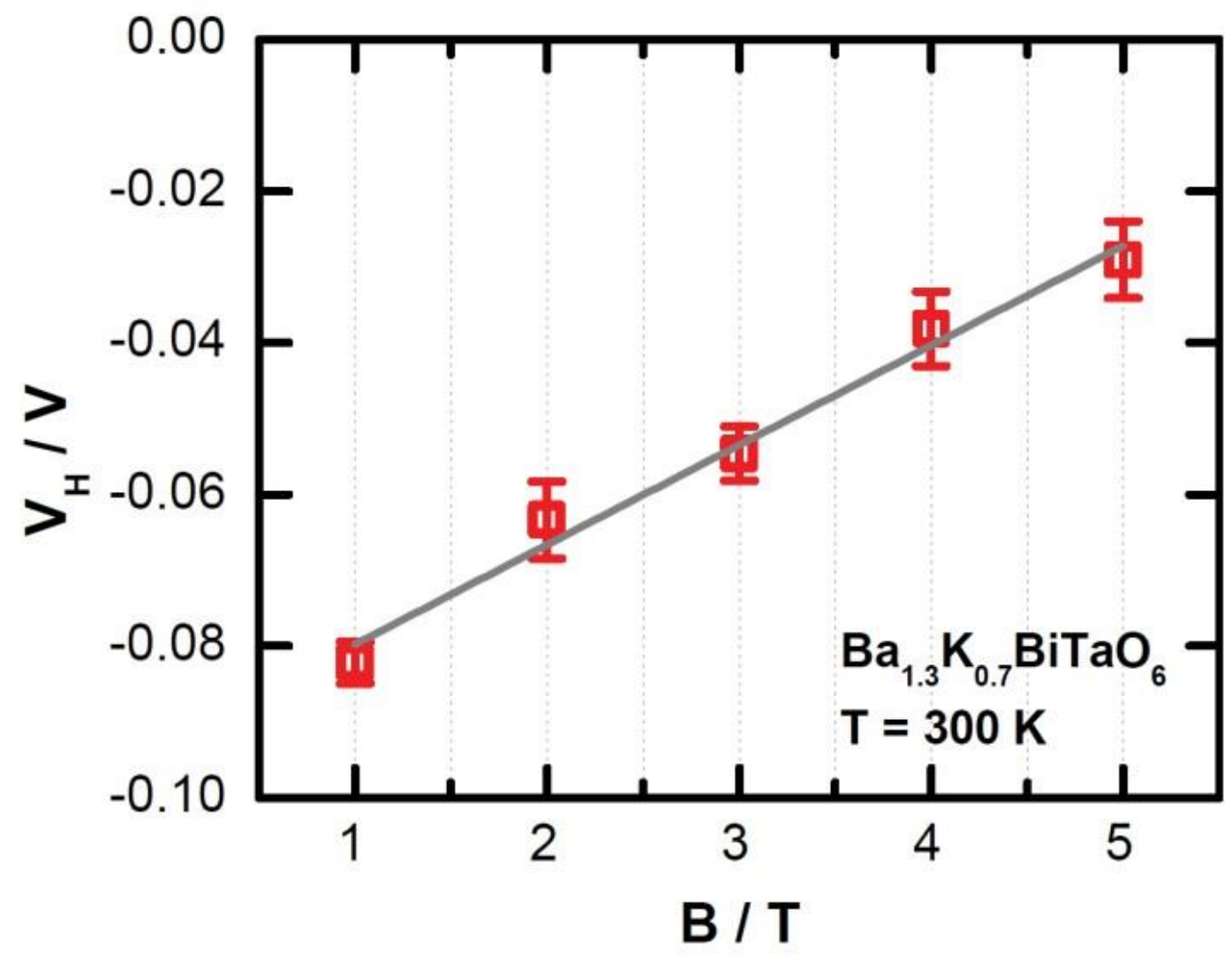

Figure 3.11 Illustrates hall voltages as a function of magnetic field. The slope was used to estimate the carrier concentration, which was calculated to be $\sim 5 \times 10^{13} \mathrm{~cm}^{-3}$ (yielding the hole mobility of $\sim 33 \mathrm{~cm}^{2} / \mathrm{V} \cdot \mathrm{s}$ ). In a separate measurement on a sample synthesized independently, the carrier concentration was calculated to be $\sim 9 \times 10^{13} \mathrm{~cm}^{-3}$ (yielding the hole mobility of $\sim 35 \mathrm{~cm}^{2} / \mathrm{V} \bullet \mathrm{s}$ ).

The result from the Hall measurement (see fig. 3.11) shows that BKBT is a p-type material. We further extract the carrier concentration from the Hall experiment, which was found to be in the range of $\sim 10^{14} \mathrm{~cm}^{-3}$. This is an astonishingly low carrier concentration given the amount of $\mathrm{K}^{+}$in the material; this level of carrier concentration has only been previously reported in high-quality single crystal works. This suggests either that we have a very low ionization ratio or that BKBT is 
fully compensated by the donor-like defects. We note that our difficulty in obtaining the p-type carrier even at high $\mathrm{K}^{+}$concentration is well known in $\mathrm{Ba}_{1-\mathrm{x}} \mathrm{K}_{\mathrm{x}} \mathrm{BiO}_{3}$, where n-type conductivity was still persistent even at $40 \% \mathrm{~K}$ substitution $\left(\mathrm{Ba}_{0.58} \mathrm{~K}_{0.42} \mathrm{BiO}_{6}\right)^{53,54}$. Likely, this suggests the need for a future study to identify the nature of the compensating defects in the bismuth perovskite oxides. From the pellet measurement, we use the Hall carrier concentration to estimate the hole mobility, which we found to be in excess of $30 \mathrm{~cm}^{2} / \mathrm{V} \cdot \mathrm{s}$. This measured mobility is higher than the value measured previously for $\mathrm{Ba}_{0.58} \mathrm{~K}_{0.42} \mathrm{BiO}_{6}$, where the mobility was found to be $\sim 2 \mathrm{~cm} / \mathrm{V} \cdot \mathrm{s}$, a result of the heavier electron being the dominant carrier in this system. Combining the mobility data with the optical gap, our BKBT compound represents the highest Hall hole mobility for a ptype transparent oxide with full range of visible transparency to our knowledge.

We point out that this preliminary value is likely an underestimation for BBT; we believe that significantly higher hole mobility can be attained with a more dense, single-crystal BBT film. However, to utilize the transport of BBT at the thin film level, methods for ionizing the dopants or overcoming the 'hole-killing' defects are essential. At present, the ionization ratio of $\mathrm{K}^{+}$in our unoptimized BBT is nearly zero, which suggests that either $\mathrm{K}^{+}$is not a good dopant or that we are limited the formation of the 'hole-killing' defects that counter our $\mathrm{K}^{+}$substitution. Although it is difficult to predict if BKBT will be able to reach the high carrier concentrations needed for "passive" transparent conducting oxides, the exceptional transmission and mobility properties justify a more careful attention, especially for applications such as thin film transistor (TFT) for which the mobility could play an important role. 


\section{Chapter 4 - Conclusion and Future Work}

\subsection{Conclusion}

In summary, we have reported on a new chemistry of high-mobility, visibly transparent p-type oxide: $\mathrm{Ba}_{2} \mathrm{BiTaO}_{6}$. Our aim was the experimental verification of predictions made using highthroughput computational screening for identification of high mobility and wide band gap p-type oxides. Experimental characterizations show $\mathrm{Ba}_{2} \mathrm{BiTaO}_{6}$ to have $>90 \%$ high transmission in the visible and the highest reported p-type mobility for a transparent oxide. To our knowledge, this is the first reported $\mathrm{Bi}$ 6s p-type transparent compound, joining $\mathrm{Bi}^{3+}$ to the selected club of elements leading to p-type transparent oxides ( $\mathrm{such}$ as $\mathrm{Sn}^{2+}, \mathrm{Cu}^{1+}$ and $\mathrm{Ag}^{1+}$ ). Although still limited by the carrier concentration, the exceptional performance of $\mathrm{Ba}_{2} \mathrm{BiTaO}_{6}$ is already evident in our preliminary characterizations. We further point out that $\mathrm{Ba}_{2} \mathrm{BiTaO}_{6}$ has a unique draw of having a ubiquitous perovskite structure, which offers a wide range of possibility of integrations with other functional perovskite materials. Our work shows how the "needle in a haystack problem" of materials discovery can be accelerated using high-throughput material computations, and how material informatics can help guide the experimental realization of new technological materials. 


\subsection{Future work}

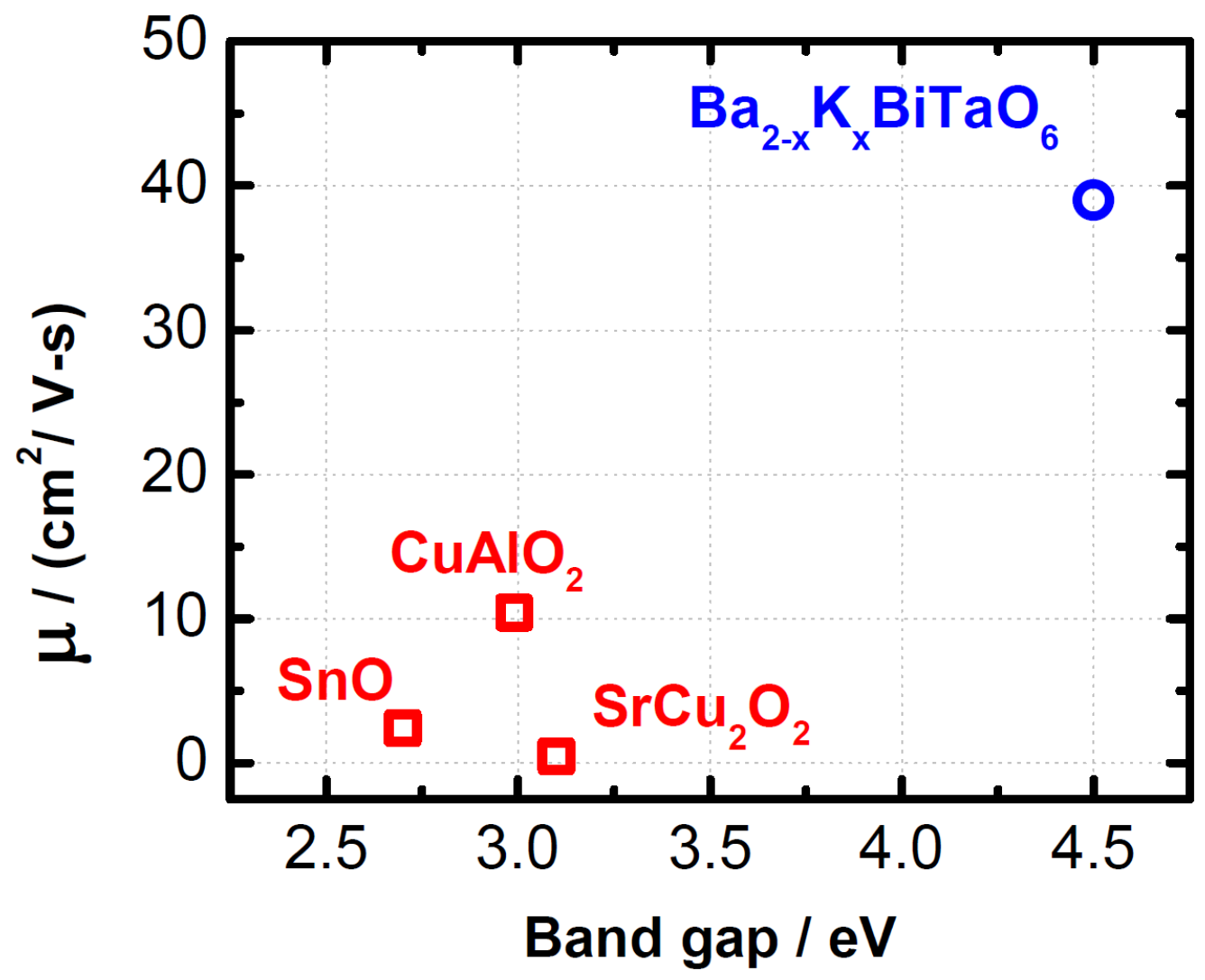

Figure 4.1 Illustrates hall mobility $\left(\mathrm{cm}^{2} / \mathrm{V}\right.$-s) vs band gap $(\mathrm{eV})$ of $\mathrm{Ba}_{2} \mathrm{BiTaO}_{6}$ in comparison with other existing state-of-the-art p-type transparent conducting oxides.

Figure 4.1 shows the comparison of hall mobility of holes and band gap of BBT vs state-of-theart existing p-type TCOs. As proved by our optical characterization (transparency, absorption data) and transport measurements, BBT is a special material with exceptionally high hole mobility and wide band gap. However, this thesis represents just the first step to understand this special material. Having said that, as discussed BBT is still limited by the charge carrier concentration, responsible for bringing down its conductivity. We predict this unexpectedly exceptionally low charge carrier 
concentration is attributed to the large number of "hole killers" or charge compensation effects that take place in the pure system.

Our next step would be to identify and quantify these defect carriers responsible for "killing" the holes. Once identified, appropriate steps should be taken to optimize the synthesis thereby trying to minimize the hole compensation effects. It would also be interesting to quantify the concentration of $\mathrm{K}$ doping in the system, whether it is same as we expect it to be. If yes, does it result in complete ionization leading to formation of extra holes as intended? As mentioned earlier, we feel hole mobility of $\sim 30 \mathrm{~cm}^{2} / \mathrm{V}$-s, even though the best known till date for a wide band gap transparent p-type semiconductor, is still an under-estimation for BBT and much higher values can be obtained for epitaxially deposited single-crystalline BBT films. 


\section{Bibliography}

1. Nomura, K. et al. Thin-film transistor fabricated in single-crystalline transparent oxide semiconductor. Science 300, 1269-72 (2003).

2. Morkoç, H. et al. Large-band-gap SiC, III-V nitride, and II-VI ZnSe-based semiconductor device technologies. J. Appl. Phys. 76, 1363 (1994).

3. Handbook of Transparent Conductors. (Springer US, 2011). doi:10.1007/978-1-44191638-9

4. Ellmer, K. Past achievements and future challenges in the development of optically transparent electrodes. Nat. Photonics 6, 809-817 (2012).

5. Izaki, M. et al. Electrochemically constructed $\mathrm{p}-\mathrm{Cu}_{2} \mathrm{O} / \mathrm{n}-\mathrm{ZnO}$ heterojunction diode for photovoltaic device. J. Phys. D. Appl. Phys. 40, 3326-3329 (2007).

6. $\mathrm{Hu}, \mathrm{S}$. et al. Amorphous $\mathrm{TiO}_{2}$ coatings stabilize $\mathrm{Si}, \mathrm{GaAs}$, and $\mathrm{GaP}$ photoanodes for efficient water oxidation. Science 344, 1005-9 (2014).

7. Ogo, Y. et al. p-channel thin-film transistor using p-type oxide semiconductor, SnO. Appl. Phys. Lett. 93, 032113 (2008).

8. Fortunato, E. et al. Transparent p-type $\mathrm{SnO}_{\mathrm{x}}$ thin film transistors produced by reactive $\mathrm{rf}$ magnetron sputtering followed by low temperature annealing. Appl. Phys. Lett. 97, 2-5 (2010).

9. Ohta, H. et al. Highly electrically conductive indium-tin-oxide thin films epitaxially grown on yttria-stabilized zirconia (100) by pulsed-laser deposition. Appl. Phys. Lett. 76, 27402742 (2000).

10. Look, D. C., Droubay, T. C. \& Chambers, S. A. Stable highly conductive ZnO via reduction of Zn vacancies. Appl. Phys. Lett. 101, (2012).

11. Carcia, P. F., McLean, R. S., Reilly, M. H. \& Nunes, G. Transparent ZnO thin-film transistor fabricated by rf magnetron sputtering. Appl. Phys. Lett. 82, 1117 (2003).

12. Bathia, A. et al. High-Mobility Bismuth-based Transparent P-Type Oxide from Highthroughput Material Screening. (2014). at <http://arxiv.org/abs/1412.4429>

13. Stauber, R. E. Thin Film Growth of Transparent p-type $\mathrm{CuAlO}_{2}$. Electrochem. Solid-State Lett. 2, 654 (1999).

14. Tonooka, K., Bando, H. \& Aiura, Y. Photovoltaic effect observed in transparent $\mathrm{p}-\mathrm{n}$ heterojunctions based on oxide semiconductors. Thin Solid Films 445, 327-331 (2003). 
15. Kudo, A. et al. Fabrication of transparent $\mathrm{p}-\mathrm{n}$ heterojunction thin film diodes based entirely on oxide semiconductors. Appl. Phys. Lett. 75, 2851 (1999).

16. Ohta, H. et al. Fabrication and photoresponse of a pn-heterojunction diode composed of transparent oxide semiconductors, p-NiO and n-ZnO. Appl. Phys. Lett. 83, 1029 (2003).

17. Nomura, K. et al. Room-temperature fabrication of transparent flexible thin-film transistors using amorphous oxide semiconductors. Nature 432, 488-92 (2004).

18. Kawazoe, H., Yanagi, H., Ueda, K. \& Hosono, H. Transparent p-Type Conducting Oxides: Design and Fabrication of p-n Heterojunctions. MRS Bulletin 25, 28-36 (2000).

19. Hautier, G., Miglio, A., Ceder, G., Rignanese, G.-M. \& Gonze, X. Identification and design principles of low hole effective mass p-type transparent conducting oxides. Nat. Commun. 4, 2292 (2013).

20. Khan, A. Growth and characterization of P-type transparent conducting oxide thin films by MOCVD. (2011). at <https://tel.archives-ouvertes.fr/tel-00582901>

21. Pellicer-Porres, J. et al. On the band gap of CuAlO2 delafossite. Appl. Phys. Lett. 88, (2006).

22. BANERJEE, A., GHOSH, C. \& CHATTOPADHYAY, K. Effect of excess oxygen on the electrical properties of transparent p-type conducting CuAlO thin films. Solar Energy Materials and Solar Cells 89, 75-83 (2005).

23. Tate, J. et al. Origin of p-type conduction in single-crystal CuAlO2. Physical Review B 80, (2009).

24. Yanagi, H. et al. Electronic structure and optoelectronic properties of transparent p-type conducting CuAlO2. J. Appl. Phys. 88, 4159-4163 (2000).

25. Kawazoe, H. et al. P-type electrical conduction in transparent thin films of CuAlO2. Nature 389, 939-942 (1997).

26. Suntivich, J. \& Hong, W. Estimating Hybridization of Transition Metal and Oxygen States in Perovskites from OK-edge X-ray Absorption Spectroscopy. J. (2014).

27. Kawazoe, H., Yasukawa, M. \& Hyodo, H. P-type electrical conduction in transparent thin films of CuAlO2. Nature (1997).

28. Look, D. C. \& Claflin, B. P-type doping and devices based on ZnO. Phys. status solidi 241, 624-630 (2004).

29. Walsh, A. \& Payne, D. Stereochemistry of post-transition metal oxides: revision of the classical lone pair model. Chem. Soc. Rev. (2011). 
30. Ogo, Y., Hiramatsu, H. \& Nomura, K. Tin monoxide as an s-orbital-based p-type oxide semiconductor: Electronic structures and TFT application. status solidi (2009).

31. Quackenbush, N. \& Allen, J. Origin of the Bipolar Doping Behavior of SnO from X-ray Spectroscopy and Density Functional Theory. Chem.(2013).

32. Allen, J., Scanlon, D., Piper, L. \& Watson, G. Understanding the defect chemistry of tin monoxide. J. Mater. Chem. C (2013).

33. Wang, H. et al. Synthesis, structure, and characterization of the series $\mathrm{BaBi}(1-\mathrm{x}) \mathrm{Ta}(\mathrm{x}) \mathrm{O}(3)$ $(0<\mathrm{or}=\mathrm{x}<\mathrm{or}=0.5)$. Inorg. Chem. 49, 5262-70 (2010).

34. Wallwork, K. S., Kennedy, B. J., Zhou, Q., Lee, Y. \& Vogt, T. Pressure and temperaturedependent structural studies of $\mathrm{Ba}_{2} \mathrm{BiTaO}_{6}$. Journal of Solid State Chemistry 178, 207-211 (2005).

35. Zhou, Q. \& Kennedy, B. J. High temperature structural studies of $\mathrm{Ba}_{2} \mathrm{BiTaO}_{6}$. Solid State Sci. 7, 287-291 (2005).

36. Baumert, B. A. Barium potassium bismuth oxide: A review. Journal of Superconductivity 8, 175-181 (1995).

37. Hinks, D., Dabrowski, B. \& Jorgensen, J. Synthesis, structure and superconductivity in the $\mathrm{Ba}_{1-} \mathrm{K}_{\mathrm{x}} \mathrm{BiO}_{3-\mathrm{y}}$ system. Nature (1988).

38. Schneemeyer, L., Thomas, J. \& Siegrist, T. Growth and structural characterization of superconducting $\mathrm{Ba}_{1-\mathrm{x}} \mathrm{K}_{\mathrm{x}} \mathrm{BiO}_{3}$ single crystals. Nature (1988).

39. Tseng, D. \& Ruckenstein, E. Structure and superconductivity of $\mathrm{BaBiO}_{3}$ doped with alkali ions. J. Mater. Res. (1990).

40. Wang, Y., Feng, G., Sutto, T. \& Shao, Z. Dielectric function of $\mathrm{BaBiO}_{3}$ investigated by electron-energy-loss spectroscopy and ellipsometry. Phys. Rev. B (1991).

41. Federici, J., Greene, B., Hartford, E. \& Hellman, E. Optical characterization of excited states in $\mathrm{BaBiO}_{3}$. Phys. Rev. B (1990).

42. Ginley, D. S. \& Perkins, J. D. in Handbook of transparent conductors 1-25 (2011). doi:10.1007/978-1-4419-1638-9

43. Jain, A., Ong, S., Hautier, G. \& Chen, W. Commentary: The Materials Project: A materials genome approach to accelerating materials innovation. APL (2013).

44. Hautier, G. \& Miglio, A. How does chemistry influence electron effective mass in oxides? A high-throughput computational analysis. Chem. (2014). 
45. Maintz, S., Deringer, V. L., Tchougréeff, A. L. \& Dronskowski, R. Analytic projection from plane-wave and PAW wavefunctions and application to chemical-bonding analysis in solids. J. Comput. Chem. 34, 2557-67 (2013).

46. Deringer, V. L., Tchougréeff, A. L. \& Dronskowski, R. Crystal orbital Hamilton population (COHP) analysis as projected from plane-wave basis sets. J. Phys. Chem. A 115, 5461-6 (2011).

47. Dronskowski, R. \& Bloechl, P. E. Crystal orbital Hamilton populations (COHP): energyresolved visualization of chemical bonding in solids based on density-functional calculations. J. Phys. Chem. 97, 8617-8624 (1993).

48. Kresse, G. \& Furthmüller, J. Efficient iterative schemes for ab initio total-energy calculations using a plane-wave basis set. Phys. Rev. B (1996).

49. Perdew, J., Burke, K. \& Ernzerhof, M. Generalized gradient approximation made simple. Phys. Rev. Lett. (1996).

50. Gonze, X., Amadon, B. \& Anglade, P. ABINIT: First-principles approach to material and nanosystem properties. Comput. Phys. (2009).

51. Ong, S., Richards, W. \& Jain, A. Python Materials Genomics (pymatgen): A robust, opensource python library for materials analysis. Comput. Mater. (2013).

52. Robertson, J. \& Clark, S. J. Limits to doping in oxides. Phys. Rev. B 83, 075205 (2011).

53. Minami, H. \& Uwe, H. Electrical Conductivity of the Oxide Superconductor $\mathrm{Ba}_{0.58} \mathrm{~K}_{0.42} \mathrm{BiO}_{2.96}$. J. Phys. Soc. Japan (1997).

54. Lee, S., Wei, J., Tang, H. \& Chien, T. Hall effect and resistivity in metallic $\mathrm{Ba}_{1-}{ }_{x} \mathrm{~K}_{x} \mathrm{BiO}_{3}$ single crystals: Absence of $1 / \mathrm{T}$ dependence in $\mathrm{R} \mathrm{h}$ and linear-to-quadratic evolution of $\varrho$ (T). Phys. C (1993). 